\title{
Structural Properties of Potts Model Partition Functions and Chromatic Polynomials for Lattice Strips
}

\author{
Shu-Chiuan $\operatorname{Chang}^{(a) *}$ and $\operatorname{Robert}^{\operatorname{Shrock}^{(a, b) * *}}$ \\ (a) C. N. Yang Institute for Theoretical Physics \\ State University of New York \\ Stony Brook, N. Y. 11794-3840 \\ (b) Physics Department \\ Brookhaven National Laboratory \\ Upton, NY 11973-5000
}

\begin{abstract}
The $q$-state Potts model partition function (equivalent to the Tutte polynomial) for a lattice strip of fixed width $L_{y}$ and arbitrary length $L_{x}$ has the form $Z(G, q, v)=\sum_{j=1}^{N_{Z, G, \lambda}} c_{Z, G, j}\left(\lambda_{Z, G, j}\right)^{L_{x}}$, where $v$ is a temperature-dependent variable. The special case of the zero-temperature antiferromagnet $(v=-1)$ is the chromatic polynomial $P(G, q)$. Using coloring and transfer matrix methods, we give general formulas for $C_{X, G}=\sum_{j=1}^{N_{X, G, \lambda}} c_{X, G, j}$ for $X=Z, P$ on cyclic and Möbius strip graphs of the square and triangular lattice. Combining these with a general expression for the (unique) coefficient $c_{Z, G, j}$ of degree $d$ in $q$ : $c^{(d)}=U_{2 d}\left(\frac{\sqrt{q}}{2}\right)$, where $U_{n}(x)$ is the Chebyshev polynomial of the second kind, we determine the number of $\lambda_{Z, G, j}$ 's with coefficient $c^{(d)}$ in $Z(G, q, v)$ for these cyclic strips of width $L_{y}$ to be $n_{Z}\left(L_{y}, d\right)=(2 d+1)\left(L_{y}+d+1\right)^{-1}\left(\begin{array}{c}2 L_{y} \\ L_{y}-d\end{array}\right)$ for $0 \leq d \leq L_{y}$ and zero otherwise. For both cyclic and Möbius strips of these lattices, the total number of distinct eigenvalues $\lambda_{Z, G, j}$ is calculated to be $N_{Z, L_{y}, \lambda}=\left(\begin{array}{c}2 L_{y} \\ L_{y}\end{array}\right)$. Results are also presented for the analogous numbers $n_{P}\left(L_{y}, d\right)$ and $N_{P, L_{y}, \lambda}$ for $P(G, q)$. We find that $n_{P}\left(L_{y}, 0\right)=n_{P}\left(L_{y}-1,1\right)=M_{L_{y}-1}$ (Motzkin number), $n_{Z}\left(L_{y}, 0\right)=C_{L_{y}}$ (the Catalan number), and give an exact expression for $N_{P, L_{y}, \lambda}$. Our results for $N_{Z, L_{y}, \lambda}$ and $N_{P, L_{y}, \lambda}$ apply for both the cyclic and Möbius strips of both the square and triangular lattices; we also point out the interesting relations $N_{Z, L_{y}, \lambda}=2 N_{D A, t r i, L_{y}}$ and $N_{P, L_{y}, \lambda}=2 N_{D A, s q, L_{y}}$, where $N_{D A, \Lambda, n}$ denotes the number of directed lattice animals on the lattice $\Lambda$. We find the asymptotic growths $N_{Z, L_{y}, \lambda} \sim L_{y}^{-1 / 2} 4^{L_{y}}$ and $N_{P, L_{y}, \lambda} \sim L_{y}^{-1 / 2} 3^{L_{y}}$ as $L_{y} \rightarrow \infty$. Some general geometric identities for Potts model partition functions are also presented.
\end{abstract}

*email: shu-chiuan.chang@sunysb.edu

**(a): permanent address; email: robert.shrock@sunysb.edu 


\section{Introduction}

The $q$-state Potts model has served as a valuable model for the study of phase transitions and critical phenomena $[1,2]$. On a lattice, or, more generally, on a (connected) graph $G$, at temperature $T$, this model is defined by the partition function

$$
Z(G, q, v)=\sum_{\left\{\sigma_{n}\right\}} e^{-\beta \mathcal{H}}
$$

with the (zero-field) Hamiltonian

$$
\mathcal{H}=-J \sum_{\langle i j\rangle} \delta_{\sigma_{i} \sigma_{j}}
$$

where $\sigma_{i}=1, \ldots, q$ are the spin variables on each vertex $i \in G ; \beta=\left(k_{B} T\right)^{-1}$; and $\langle i j\rangle$ denotes pairs of adjacent vertices. The graph $G=G(V, E)$ is defined by its vertex set $V$ and its edge set $E$; we denote the number of vertices of $G$ as $n=n(G)=|V|$ and the number of edges of $G$ as $e(G)=|E|$. We use the notation

$$
K=\beta J, \quad a=u^{-1}=e^{K}, \quad v=a-1
$$

so that the physical ranges are (i) $a \geq 1$, i.e., $v \geq 0$ corresponding to $\infty \geq T \geq 0$ for the Potts ferromagnet, and (ii) $0 \leq a \leq 1$, i.e., $-1 \leq v \leq 0$, corresponding to $0 \leq T \leq \infty$ for the Potts antiferromagnet. One defines the (reduced) free energy per site $f=-\beta F$, where $F$ is the actual free energy, via

$$
f(\{G\}, q, v)=\lim _{n \rightarrow \infty} \ln \left[Z(G, q, v)^{1 / n}\right] .
$$

where we use the symbol $\{G\}$ to denote $\lim _{n \rightarrow \infty} G$ for a given family of graphs.

Let $G^{\prime}=\left(V, E^{\prime}\right)$ be a spanning subgraph of $G$, i.e. a subgraph having the same vertex set $V$ and an edge set $E^{\prime} \subseteq E$. Then $Z(G, q, v)$ can be written as the sum [3]-[6]

$$
\begin{aligned}
Z(G, q, v) & =\sum_{G^{\prime} \subseteq G} q^{k\left(G^{\prime}\right)} v^{e\left(G^{\prime}\right)} \\
& =\sum_{r=k(G)}^{n(G)} \sum_{s=0}^{e(G)} z_{r s} q^{r} v^{s}
\end{aligned}
$$

where $k\left(G^{\prime}\right)$ denotes the number of connected components of $G^{\prime}$ and $z_{r s} \geq 0$. Since we only consider connected graphs $G$, we have $k(G)=1$. The formula (1.5) shows that $Z(G, q, v)$ is a polynomial in $q$ and $v$ and enables one to generalize $q$ from $\mathbb{Z}_{+}$to $\mathbb{R}_{+}$and, indeed, to $\mathbb{C}$. The Potts model partition function $Z(G, q, v)$ on a graph $G$ is essentially equivalent to the Tutte polynomial [7]-[9] $T(G, x, y)$ and Whitney rank polynomial $R(G, \xi, \eta)[2],[4],[10]$ for this graph, as discussed in the appendix. 
One special case of the Potts model partition function that is of particular interest is the zero-temperature limit of the Potts antiferromagnet (AF). For sufficiently large $q$, on a given lattice or graph $G$, this exhibits nonzero ground state entropy (without frustration). This is equivalent to a ground state degeneracy per site (vertex), $W>1$, since $S_{0}=k_{B} \ln W$. The $T=0$ (i.e., $v=-1$ ) partition function of the above-mentioned $q$-state Potts antiferromagnet (PAF) on $G$ satisfies

$$
Z(G, q,-1)=P(G, q)
$$

where $P(G, q)$ is the chromatic polynomial (in $q$ ) expressing the number of ways of coloring the vertices of the graph $G$ with $q$ colors such that no two adjacent vertices have the same color $[3,10,12,13]$. This is termed a proper vertex coloring of $G$. The minimum number of colors necessary for this coloring is the chromatic number of $G$, denoted $\chi(G)$. Thus ${ }^{1}$

$$
W(\{G\}, q)=\lim _{n \rightarrow \infty} P(G, q)^{1 / n} .
$$

A second special case of the Potts model partition function is for infinite temperature, i.e., $K=0$ or equivalently $v=0$. In this case, as is clear from either (1.1) with (1.2) or from (1.5), $Z$ reduces to the single term

$$
Z(G, q, 0)=q^{n(G)}
$$

A third special case is the Potts ferromagnet in the limit of zero temperature, $T \rightarrow 0$, i.e., $v \rightarrow \infty$.

Here we shall consider families of graphs that are cyclic strips of the square and triangular lattices, taken to be oriented horizontally, with free transverse boundary conditions, denoted $F B C_{y}$, and periodic longitudinal boundary conditions, denoted $P B C_{x}$. A given strip of the triangular lattice may be visualized by starting with the corresponding strip of the square lattice and adding diagonal bonds joining, say, the upper left to lower right vertices of each square. The length and width are taken to be $L_{x}$ and $L_{y}$ vertices, and the total number of vertices is $n=L_{x} L_{y}$. We shall also make some comments about the analogous strips with twisted periodic longitudinal, i.e., Möbius, boundary conditions, denoted $T P B C_{x}$ or $M b$. These various families of lattice strip graphs are examples of recursive families of graphs, in the sense that a strip of length $L_{x}+1$ is constructed by the addition of a given subgraph (here, a transverse layer of the strip) to the strip of length $L_{x}$. As derived in $[15,16]$, using a type of transfer matrix argument, a general form for the Potts model partition function for the strip graphs considered here, or more generally, for a recursively defined graph comprised of $L_{x}=m$ repeated subunits (transverse layers of the strip here), is

$$
Z(G, q, v)=\sum_{j=1}^{N_{Z, G, \lambda}} c_{Z, G, j}\left(\lambda_{Z, G, j}\right)^{m}
$$

\footnotetext{
${ }^{1}$ At certain special points $q_{s}$ (typically $q_{s}=0,1, \ldots, \chi(G)$ ), one has the noncommutativity of limits $\lim _{q \rightarrow q_{s}} \lim _{n \rightarrow \infty} P(G, q)^{1 / n} \neq \lim _{n \rightarrow \infty} \lim _{q \rightarrow q_{s}} P(G, q)^{1 / n}$, and hence it is necessary to specify the order of the limits in the definition of $W\left(\{G\}, q_{s}\right)[14]$.
} 
where $\lambda_{Z, G, j}$ is a function of $q$ and $v$, and both $\lambda_{Z, G, j}$ and the coefficients $c_{Z, G, j}$ are independent of $L_{x}$. The coefficients $c_{Z, G, j}$ are functions only of $q$ and, indeed, are polynomials, for the cyclic strip graphs of interest here. (We shall comment on other types of strip graphs below.)

From (1.6), it also follows that the chromatic polynomial has the same structure,

$$
P(G, q)=\sum_{j=1}^{N_{P, G, \lambda}} c_{P, G, j}\left(\lambda_{P, G, j}\right)^{m}
$$

where $N_{P, G, \lambda}$ depends on $G ; c_{P, G, j}$ and $\lambda_{P, G, j}$ are independent of $L_{x}=m$; and

$$
N_{P, G, \lambda} \leq N_{Z, G, \lambda}
$$

As will be shown below, for the strips under consideration here, this inequality is realized as an equality for the circuit graph $C_{n}$ and as a strict inequality for widths $L_{y} \geq 2$ (see Tables 1 and 3). For the same reason, it follows that when one sets $v=-1$ in $Z(G, q, v)$, i.e. specializes to the $T=0$ Potts antiferromagnet, a certain number $N_{Z, G, \lambda}-N_{P, G, \lambda}$ of the $\lambda_{Z, G, j}$ 's vanish and the remaining, nonvanishing $\lambda_{Z, G, j}(q, v)$ 's are precisely those occurring in $P(G, q)$ :

$$
\text { If } \lambda_{Z, G, j}(q, v=-1) \neq 0 \text { then } \lambda_{Z, G, j}(q,-1)=\lambda_{P, G, j}(q) .
$$

Previous calculations of chromatic polynomials for recursive families of graphs of arbitrary length include [18]-[49]; we shall also use the results of calculations of Potts model partition functions (Tutte polynomials) for cyclic and Möbius strip graphs of arbitrary length in $[15,16,47]$. Some relevant results on transfer matrices and the Temperley-Lieb algebra are in $[29],[50]-[53]$.

Several basic questions about the structure of $Z(G, q, v)$ and $P(G, q)$ for these cyclic strip graphs are the following:

1. Can one obtain a general formula for the coefficients $c_{Z, G, j}$ and $c_{P, G, j}$ in (1.9) and $(1.10) ?$

2. What are the respective sums of the coefficients $c_{Z, G, j}$ and $c_{P, G, j}$ in (1.9) and (1.10)?

3. For a given cyclic strip $G$, how many $\lambda_{Z, G, j}$ 's in (1.9) have a particular coefficient, and how many $\lambda_{P, G, j}$ 's in (1.10) have this coefficient?

4. What are the total numbers of different terms $N_{Z, G, \lambda}$ and $N_{P, G, \lambda}$ in (1.9) and (1.10)?

In this paper, we shall obtain a general formula for the coefficients $c_{Z, G, j}$ and $c_{P, G, j}$, an answer to the second pair of questions, and, based on these results, answers to the remaining two pairs of questions. We shall also comment on other types of lattice strips.

The chromatic polynomial, can be calculated by various methods, including iterative application of the deletion-contraction theorem (e.g., [10]), a certain matrix technique [21], 
a generating function method [33, 40], or a coloring (compatibility) matrix method [22, 43]. Similarly, the Potts model partition function or equivalent Tutte polynomial can be calculated by iterative application of the generalized deletion-contraction theorem [10] or by transfer matrix methods including a relation with the Temperley-Lieb algebra [29, 51, 53]. Of course these different methods can be used to provide cross-checks; for example, iterated deletion-contraction and transfer matrix methods were used to check each other in [16, 47] and deletion-contraction and coloring matrix methods were used together in works such as [45]. The last, the coloring matrix method, is also useful for deriving rigorous upper and lower bounds on $W(\{G\}, q)[22],[23],[24]$. In this method, one first selects a transverse slice of the strip, denoted $\mathcal{L}_{L_{y}}$. For our cyclic graphs, this is simply a line graph with $L_{y}$ vertices (oriented vertically, given that we orient the long direction of the strip horizontally). Denote an allowed $q$-coloring of this path as $c\left(\mathcal{L}_{L_{y}}\right)$. The number of allowed colorings of the path $\mathcal{L}_{L_{y}}$ is $\mathcal{N}=P\left(\mathcal{L}_{L_{y}}, q\right)$. For our chromatic polynomials of cyclic strips of the square and triangular lattices, $P\left(\mathcal{L}_{n}, q\right)=P\left(T_{L_{y}}, q\right)$, where $T_{n}$ is the tree graph with $n$ vertices and

$$
P\left(T_{n}, q\right)=q(q-1)^{n-1} .
$$

Now focus on two adjacent paths $\mathcal{L}_{L_{y}}$ and $\mathcal{L}_{L_{y}}^{\prime}$. Define compatible $q$-colorings of these paths as colorings such that no two adjacent vertices $v \in \mathcal{L}_{L_{y}}$ and $v^{\prime} \in \mathcal{L}_{L_{y}}^{\prime}$ (i.e. vertices connected by an edge = bond of the lattice strip graph) have the same color. One can then associate with this pair of paths an $\mathcal{N} \times \mathcal{N}$ dimensional symmetric matrix $\mathcal{T}$ with entries $\mathcal{T}_{c\left(\mathcal{L}_{L_{y}}\right), c\left(\mathcal{L}_{L_{y}}^{\prime}\right)}=1$ or 0 if the $q$-colorings of $\mathcal{L}_{L_{y}}$ and $\mathcal{L}_{L_{y}}^{\prime}$ are or are not compatible, respectively. Then the chromatic polynomial of the cyclic strip of the lattice $\Lambda$, taken here to be square $(s q)$ or triangular $(t r i)$, is (with $L_{x}=m$ ) given by

$$
P\left(\Lambda, L_{y} \times L_{x}, F B C_{y}, P B C_{x}, q\right)=\operatorname{Tr}\left(\mathcal{T}^{m}\right) .
$$

Since $\mathcal{T}$ is a symmetric real matrix (indeed, composed only of 0 's and 1's, although we do not need this here), it can be diagonalized by an orthogonal transformation, so that the above trace is $\operatorname{Tr}\left(\mathcal{T}^{m}\right)=\sum_{j}\left(\lambda_{P, G, j}\right)^{m}$, where the $\lambda_{P, G, j}$ 's are the eigenvalues of $\mathcal{T}$. In the context of the $q$-coloring problem, if one denotes the multiplicity of the $j$ 'th distinct eigenvalue by $c_{P, G, j}$, one obtains the formula (1.10). That is, from the coloring matrix viewpoint, $c_{P, G, j}$ is the dimension of the invariant subspace in the full $\mathcal{N}$-dimensional space of coloring configurations of the transverse slice of the strip corresponding to the eigenvalue $\lambda_{P, G, j}$. For the full temperature-dependent Potts model partition function, one can define an analogous matrix, $\mathcal{T}_{Z}$ whose entries, rather than being 0 and 1 , are appropriate Boltzmann weights for the given spin configurations on the successive transverse slices $\mathcal{L}_{L_{y}}$ and $\mathcal{L}_{L_{y}}^{\prime}$. Then this partition function is (with $L_{x}=m$ )

$$
Z\left(\Lambda, L_{y} \times L_{x}, F B C_{y}, P B C_{x}, q, v\right)=\operatorname{Tr}\left[\left(\mathcal{T}_{Z}\right)^{m}\right]
$$

which was used in $[15,16]$ to derive the formula (1.9). Note that in this case all colorings, including those that yield the same color on adjacent vertices, are allowed. One can obtain these formulas (1.14) and (1.15) for sufficiently large integral $q$ that the multiplicities $c_{P, G, j}$ 
and $c_{Z, G, j}$ are positive integers; in both of these cases, the fact that the coefficients are multiplicities shows that for sufficiently large positive integer $q$ they are also positive integers. In the case of the chromatic polynomial, it is obvious that $c_{P, G, j}$ can depend only on $q$; in the case of the full Potts model partition function, for the cyclic strip graphs considered here, this also follows from (1.15) and (1.9), since the multiplicities of the eigenvalues cannot depend on the variable parameter $v \in(-1, \infty)$.

Note that, while the coefficients $c_{P, G, j}$ and $c_{Z, G, j}$ can be obtained as multiplicities of the distinct eigenvalues $\lambda_{P, G, j}$ and $\lambda_{Z, G, j}$ for sufficiently large integer $q$, when one considers positive $q<4$, they may be zero or negative (see eqs. (2.17)-(2.21) below). More generally, while they play the role of eigenvalue multiplicities for sufficiently large integer $q$, the domain of their definition may be generalized via (1.5) to $q \in \mathbb{R}_{+}$or, indeed, to $q \in \mathbb{C}$. Some properties of determinants of coloring matrices for various strip graphs are given in the appendix.

The dimension of the space of coloring configurations, $\mathcal{N}$, is equal to the sum of the multiplicities of each distinct eigenvalue, i.e., the sum of the dimensions of the invariant subspaces corresponding to each of these distinct eigenvalues. For the chromatic polynomial, this is $\mathcal{N}$, which is equal to the sum

$$
C_{P, G}=\sum_{j=1}^{N_{P, G, \lambda}} c_{P, G, j}
$$

while for the full Potts model partition function, we shall denote it as

$$
C_{Z, G}=\sum_{j=1}^{N_{Z, G, \lambda}} c_{Z, G, j} .
$$

General results for these sums will be given below for the strips of interest.

Before proceeding, we note that for the chromatic polynomial, since two identical color configurations on $\mathcal{L}_{L_{y}}$ and $\mathcal{L}^{\prime}{ }_{L_{y}}$ are incompatible, the diagonal elements of the coloring matrix are zero and hence its trace is zero:

$$
\operatorname{Tr}(\mathcal{T}(G, q))=0 \quad \text { for } P(G, q)
$$

Since the coefficients $c_{P, G, j}$ are just the multiplicities of the given eigenvalues $\lambda_{P, G, j}$, we can write this as the sum over distinct $\lambda_{P, G, j}$ 's:

$$
\sum_{j=1}^{N_{P, G, \lambda}} c_{P, G, j} \lambda_{P, G, j}=0 .
$$

In the following, since we shall present results that are applicable to cyclic strips of both the square and triangular lattices, we shall often leave the lattice type $\Lambda$ and the boundary conditions $\left(F B C_{y}, P B C_{x}\right)$ implicit in the notation where they are obvious. The fact that these results will be applicable to cyclic strips of both the square and triangular lattices 
depends on the property that (having expressed the strip of the triangular lattice as a strip of the square lattice with diagonal bonds added to each square, as described above) the transverse slices, i.e., line graphs of $L_{y}$ vertices, of both the cyclic square-lattice and triangular-lattice strips are identical. ${ }^{2}$ Similarly, for quantities that are independent of some part of $G$, such as $c_{Z, G, j}$ and $\lambda_{Z, G, j}$, which are independent of $L_{x}$, this will be incorporated in the notation.

\section{Coefficients in Potts Model Partition Functions for Cyclic Lattice Strips}

In this section we address the first pair of questions posed in the Introduction. From our exact solutions of the chromatic polynomials and, more generally, of the full temperaturedependent Potts model partition functions on cyclic strips of the square and triangular lattice, we have found that the coefficients $c_{Z, G, j}$ and $c_{P, G, j}$ are polynomials in $q$. Further, we have found that for a given cyclic strip of the square or triangular lattice with width $L_{y}$, the coefficients $c_{P, G, j}$ and $c_{Z, G, j}$ are of a limited set; only one type of polynomial of each degree in $q$ occurs $^{3}$, and, denoting this as $c^{(d)}$, there are coefficients of the form $c^{(d)}$ with $0 \leq d \leq L_{y}$. We infer the following general formula for $c^{(d)}$, i.e. for the multiplicity of the corresponding eigenvalue of $\mathcal{T}_{Z}$ :

$$
c^{(d)}=U_{2 d}\left(\frac{\sqrt{q}}{2}\right)
$$

where $U_{n}(x)$ is the Chebyshev polynomial of the second kind, defined by (e.g. $\left.[54,55]\right)$

$$
\begin{aligned}
U_{n}(x) & =\frac{1}{\sqrt{1-x^{2}}} \operatorname{Im}\left[\left(x+i \sqrt{1-x^{2}}\right)^{n+1}\right] \\
& =\sum_{j=0}^{\left[\frac{n}{2}\right]}(-1)^{j}\left(\begin{array}{c}
n-j \\
j
\end{array}\right)(2 x)^{n-2 j}
\end{aligned}
$$

where in eq. (2.2) and similar equations below, the notation $\left[\frac{n}{2}\right]$ in the upper limit on the summand means the integral part of $\frac{n}{2}$. In the first line of $(2.2), x$ must be real, but the

\footnotetext{
${ }^{2}$ Although the transverse slices are identical for the cyclic strips of the square and triangular lattice, they are different for other lattices, such as the honeycomb = brick lattice, and this difference was also significant for the earlier use of coloring matrix methods to obtain rigorous bounds on the Potts model ground state degeneracy per site, $W[22]-[24]$.

${ }^{3}$ From the studies of Möbius strips of the square lattice [18, 42, 44, 48], we find that the coefficients are again polynomials, of the form $\pm c^{(d)}$; however, for Möbius strips of the triangular lattice, the coefficients are not, in general, polynomials, but algebraic functions of $q$, as was shown by the exact solutions for the chromatic polynomial in [42] and for the full Potts model partition in [47] for the $L_{y}=2$ Möbius strip. For strips with torus boundary conditions, exact solutions $[45,46]$ show that the coefficients are polynomials of degree $d$ in $q$, but it is not, in general, the case that there is a unique coefficient of degree $d$ and they are not, in general, of the form $c^{(d)}$. We shall comment further on this below.
} 
polynomial in the second line of $(2.2)$ constitutes a definition of $U_{n}(x)$ for complex $x$. The first few of these coefficients are, with $x=\frac{\sqrt{q}}{2}$,

$$
\begin{gathered}
c^{(0)}=U_{0}(x)=1 \\
c^{(1)}=U_{2}(x)=4 x^{2}-1 \\
=q-1 \\
c^{(2)}=U_{4}(x)=16 x^{4}-12 x^{2}+1 \\
=q^{2}-3 q+1 \\
c^{(3)}=U_{6}(x)=64 x^{6}-80 x^{4}+24 x^{2}-1 \\
=q^{3}-5 q^{2}+6 q-1 \\
c^{(4)}=U_{8}(x)=256 x^{8}-448 x^{6}+240 x^{4}-40 x^{2}+1 \\
q^{4}-7 q^{3}+15 q^{2}-10 q+1 \\
=(q-1)\left(q^{3}-6 q^{2}+9 q-1\right)
\end{gathered}
$$

and so forth for higher $d$. We have found, as an equivalent formula $[46,48]$

$$
c^{(d)}=\prod_{k=1}^{d}\left(q-q_{d, k}\right)
$$

where

$$
q_{d, k} \equiv 2+2 \cos \left(\frac{2 \pi k}{2 d+1}\right)=4 \cos ^{2}\left(\frac{\pi k}{2 d+1}\right), \quad \text { for } \quad k=1,2, \ldots d .
$$

Note that the apparent square root in (2.1) is absent in the actual formula (2.1) for the $c^{(d)}$ 's, which applies for arbitrary complex $q$. One way to derive (2.1) for cyclic strips is to use the fact that the partition function is given by the trace (1.15), which, with (1.9) makes clear the role of the coefficients $c_{Z, G, j}$ as multiplicities of eigenvalues of the transfer matrix $\mathcal{T}_{Z}$; then, one utilizes the connection with the Temperley-Lieb algebra to infer that these multiplicities are given by $c^{(d)}$ (for sufficiently large integer $q$ where the $c^{(d)}$ are positive integers), and finally, one continues this result to general complex $q$. Related discussions 
of dimensions of invariant subspaces of operators in the Temperley-Lieb algebra are given in [52],[53]. The form (2.8) is also related to the property that each coefficient $c^{(d)}$ has a simple zero at the Tutte-Beraha number $B_{2 d+1}$; indeed, one could derive the form (2.1) by using this as a starting point. In this approach, one would start with the property that $c^{(d)}$ has the factor $\left(q-B_{2 d+1}\right)=\left(q-q_{d, 1}\right)$. Since $B_{2 d+1}$ is, in general, irrational, it is necessary to symmetrize the product $c^{(d)}=\prod\left(q-q_{z}\right)$ over the zeros $q_{z}$ in order to obtain rational (indeed, integer) coefficients for the $c^{(d)}$. This symmetrization yields (2.8). To see this, we note that to incorporate this zero of $c^{(d)}$ at $q=B_{2 d+1}$ one can start with the identity $[\exp (2 \pi i /(2 d+1))]^{2 d+1}-1=0$, express this as $\left[\cos \left(\frac{2 \pi}{2 d+1}\right)+i \sin \left(\frac{2 \pi}{2 d+1}\right)\right]^{2 d+1}$, expand the resulting expression, and then impose the condition that the polynomial vanishes at $q=q_{d, 1}=B_{2 d+1}$ by setting $\cos (2 \pi /(2 d+1))=q / 2-1$. This actually yields two equations, one for the real part and one for the imaginary part. Concentrating on the real part, we observe that the resultant equation has the form $(1 / 2)(q-4)\left(c^{(d)}\right)^{2}$. This yields the result

$$
c^{(d)}=\left[\frac{2}{q-4}\left(T_{2 d+1}\left(\frac{q}{2}-1\right)-1\right)\right]^{1 / 2}
$$

where $T_{n}(x)$ is the Chebyshev polynomial of the first kind, defined by (e.g., $\left.[54,55]\right)$

$$
\begin{aligned}
T_{n}(x) & =\operatorname{Re}\left[\left(x+i \sqrt{1-x^{2}}\right)^{n}\right] \\
& =\frac{1}{2} \sum_{j=0}^{\left[\frac{n}{2}\right]}(-1)^{j} \frac{n}{n-j}\left(\begin{array}{c}
n-j \\
j
\end{array}\right)(2 x)^{n-2 j}
\end{aligned}
$$

As with $U_{n}(x)$, in the first line of (2.11), $x$ must be real, but the polynomial in the second line of $(2.11)$ constitutes a definition of $T_{n}(x)$ for complex $x$; note also that the right-hand side of the second line is defined to be 1 for $n=0$. Simplifying eq. (2.10), one finally obtains eq. (2.1) in terms of the Chebyshev polynomial of the second kind. The starting point is equivalent to the identity $[\exp (2 \pi k /(2 d+1))]^{2 d+1}-1=0$, and hence it follows that $c^{(d)}$ vanishes at $q_{d, k}$ for $2 \leq k \leq d$ as well as for $k=1$. This implies the formula (2.8).

The general formula (2.1) or the equivalent result (2.8) gives a deeper understanding of, the known exact solutions for chromatic polynomials and the full Potts model partition functions on cyclic strips of the square and triangular lattice. For reference, these include the degenerate case $L_{y}=1$ (circuit graph), and the cases $L_{y}=2$ in [18], $L_{y}=3$ in [42], and $L_{y}=4$ in [48] for cyclic strips of the square lattice; and the cases $L_{y}=2$ in [42] and $L_{y}=3$ and $L_{y}=4$ in [46] for cyclic strips of the triangular lattice. For the full Potts model partition functions, the exact solutions include the elementary $L_{y}=1$ solution for the circuit graph, and the $L_{y}=2$ solutions in [16] and [47] for cyclic strips of the square and triangular lattices, respectively. Explicit examples will be given below. As noted, the coefficients with degree $d$ occur for $0 \leq d \leq L_{y}$. In the following we shall work out implications of the formula (2.1) for the structure of the chromatic polynomial and full Potts model partition function for arbitrarily wide cyclic strips of the square and triangular lattices. 
From the formula (2.1) or (2.8) and properties of the Chebyshev polynomials of the second kind, one can establish a number of useful properties of the coefficients $c^{(d)}$. First, we recall that a generating function for the $U_{n}(x)$ is (e.g. [54])

$$
\frac{1}{1-2 x z+z^{2}}=\sum_{n=0}^{\infty} U_{n}(x) z^{n}
$$

The formal sum in (2.12) converges if $|x|<1$ and $|z|<1$; however, for our purposes, we shall only use it as a means of extracting the coefficient of the $z^{n}$ term as $U_{n}(x)$, and this can be done for any $x$ and $z$. We infer the following properties of the $c^{(d)}$ :

1. $c^{(d)}$ is a polynomial of degree $d$ in the variable $q$, with integer coefficients, highest-order term equal to $q^{d}$, and alternating signs for the subsequent terms of descending degree in $q$.

2. We recall the definition of a unimodal polynomial as one with the property that the magnitudes of its coefficients increase monotonically up to a point and then (with possible equality of the maximal two coefficients) decrease monotonically. From the property that the magnitudes of the coefficients of $U_{n}(x)$ are unimodal, it follows directly that the same is true of the magnitudes of the coefficients of $c^{(d)}$.

3. The zeros of $c^{(d)}$ are real, positive, simple, lie in the interval $0<q<4$ and are given as follows:

$$
c^{(d)}=0 \quad \text { at } \quad q=q_{d, k}, \quad k=1,2, \ldots, d
$$

In the limit $d \rightarrow \infty$, these zeros become dense in this interval.

4. If $d \geq 1$, then one of the zeros of $c^{(d)}$ is at a Tutte-Beraha number, namely,

$$
q_{d, 1}=B_{2 d+1}
$$

where the Tutte-Beraha number $B_{r}$ is defined as [9],[26]

$$
B_{r}=2+2 \cos \left(\frac{2 \pi}{r}\right)=4 \cos ^{2}\left(\frac{\pi}{r}\right), r=1,2, \ldots
$$

The first Tutte-Beraha numbers are $B_{1}=4, B_{2}=0, B_{3}=1, B_{4}=2, B_{5}=(3+$ $\sqrt{5}) / 2=2.618 . ., B_{6}=3$.

5. Depending on the value of $d, c^{(d)}$ may have other zero(s) at Tutte-Beraha number(s) distinct from $B_{2 d+1}$ in (2.14). This cannot happen if $2 d+1$ is prime. If $2 d+1$ is not prime, consider the case where there exists $d^{\prime}$ such that $k\left(2 d^{\prime}+1\right)=2 d+1$. Since $2 d+1$ and $2 d^{\prime}+1$ are both odd, it follows that $k$ is also odd, so we can write this equation in a symmetric manner as

$$
\left(2 d^{\prime}+1\right)\left(2 d^{\prime \prime}+1\right)=2 d+1 .
$$


Then $c^{(d)}$ has additional zero(s) at $B_{2 d^{\prime}+1}$ and $B_{2 d^{\prime \prime}+1}$. These degenerate into a single additional zero if $2 d+1=p^{2}$ where $p$ is prime. Note that the factorization (2.16) is not unique. For example, consider $c^{(d)}$ for $d=52$. Then $2 d+1$ has the full and unique factorization $105=3 \cdot 5 \cdot 7$. There are thus three ways of writing the twofold factorization in (2.16): $3 \cdot 35,5 \cdot 21$, and $7 \cdot 15$, and hence, in addition to the zero at the Tutte-Beraha number $B_{2 d+1}=B_{105}$, the coefficient $c^{(105)}$ also has zeros at the six other Tutte-Beraha numbers $B_{3}, B_{5}, B_{7}, B_{15}, B_{21}$, and $B_{35}$. As this example makes clear, in other cases there could be more than three twofold factorizations of the form (2.16).

6. As a particular case of the previous item, if $d=1 \bmod 3$, then, setting $d=3 j+1$ and substituting in the above equation, we find $\left(2 d^{\prime}+1\right)\left(2 d^{\prime \prime}+1\right)=3(2 j+1)$. If $j=0$ so that $d=1$, i.e., $c^{(d)}=q-1$, then there is only the single zero at $B_{3}=1$ given by $(2.14)$. If $j=1$, i.e., $d=4$, then $c^{(4)}$ has a zero at $B_{3}$ and at $B_{9}$. If $j \geq 2$, then in addition to the zero at $B_{2 d+1}$ given by $(2.14), c^{(d)}$ has zeros at the Tutte-Beraha numbers $B_{3}$ and $B_{2 j+1}$ (as well as possible others). Note that since $c^{(d)}$ has only simple zeros, it follows that if $d=1 \bmod 3$, then has a simple factor $(q-1)$. (The expression given above for $c^{(4)}$ illustrates this.)

7. Since $U_{2 n}(0)=(-1)^{n}$, it follows that

$$
c^{(d)}=(-1)^{d} \quad \text { for } \quad q=0
$$

8.

$$
\text { If } q=1 \text { then } c^{(d)}= \begin{cases}1 & \text { if } d=0 \bmod 3 \\ 0 & \text { if } d=1 \bmod 3 \\ -1 & \text { if } d=2 \bmod 3\end{cases}
$$

9.

$$
\text { If } q=2 \text { then } c^{(d)}= \begin{cases}1 & \text { if } d=0,1 \bmod 4 \\ -1 & \text { if } d=2,3 \bmod 4\end{cases}
$$

10.

$$
\text { If } q=3 \text { then } c^{(d)}= \begin{cases}1 & \text { if } d=0,2 \bmod 6 \\ 2 & \text { if } d=1 \bmod 6 \\ -1 & \text { if } d=3,5 \bmod 6 \\ -2 & \text { if } d=4 \bmod 6\end{cases}
$$

11.

$$
c^{(d)}=2 d+1 \text { for } q=4 \text {. }
$$

12. As a consequence of the inequality $\left|U_{n}(x)\right| \leq n+1$ for $-1 \leq x \leq 1$, we have the inequality

$$
\left|c^{(d)}\right| \leq 2 d+1 \quad \text { for } 0 \leq q \leq 4 .
$$

This inequality is saturated at $q=4$, i.e. $x=1$. 
13. Using the recursion relation for Chebyshev polynomials,

$$
U_{n+1}(x)=2 x U_{n}(x)-U_{n-1}(x)
$$

iteratively, we find the resulting recursion for the $c^{(d)}$,

$$
c^{(d+1)}=(q-2) c^{(d)}-c^{(d-1)} .
$$

If one lets

$$
q=2+2 \cos \theta=4 \cos ^{2}\left(\frac{\theta}{2}\right)
$$

one sees that the argument of the Chebyshev polynomial of the second kind in (2.1) is given by $x=\frac{\sqrt{q}}{2}=\cos (\theta / 2)$. A relevant identity is (with $\omega=\theta / 2$ here)

$$
U_{n}(\cos \omega)=\frac{\sin ((n+1) \omega)}{\sin \omega} .
$$

The fact that the transformation (2.25) applies, with $\theta$ real, for $q \in[0,4]$ shows the special role of this interval for Potts model partition functions on the strips under consideration here. This makes an intriguing connection with the 2D Potts ferromagnet, which has a second-order phase transition for the values $q=2$ (Ising), $q=3$, and $q=4$; and a first-order phase transition for $q \geq 5$. Of course, the physical thermodynamic behavior of the Potts ferromagnet on the infinite-length, finite width strips is quite different from that of the model on 2D lattices; in the former cases, since these are quasi-one-dimensional, the model has only a zero-temperature critical point rather than a finite-temperature phase transition. For the antiferromagnetic Potts model, the transformation means that the value $q=4$ is special (as the positive value of $q$ where the angle $\theta$ changes from being real to imaginary) and is in accord with the special role of $q=4$ in this model on the infinite two-dimensional lattice [29] (see also [32]). This is somewhat similar to earlier situations in which studies of lowerdimensional realizations of spin models gave insight into properties of higher-dimensional realizations (e.g. [56]-[58] for $O(N)$ models and [16, 47, 59] for Potts/Ising models). However, in assessing the connection of the special role of the value $q=4$ in the coefficients $c^{(d)}$ with the $T=0$ critical point at $q=4$ for the Potts antiferromagnet on the triangular lattice, one must also taken into account the fact that the formula (2.1) also applies for the cyclic strips of the square lattice that have been studied; however, again taking the limit $L_{y} \rightarrow \infty$, the Potts antiferromagnet on the square lattice has a zero-temperature critical point at $q=3$ rather than at $q=4[17]$.

One of the interesting aspects of the zeros $q_{d, k}$ of $c^{(d)}$ is that at these values of $q$, the critical exponents of the 2D Potts model are rational. We recall that the thermal, magnetic, and tricritical exponents for the paramagnetic to ferromagnetic transition in the $2 \mathrm{D} q$-state Potts ferromagnet are, for $q \leq 4$, [60]-[62],[2]

$$
y_{t}=\frac{3(1-u)}{2-u}
$$




$$
y_{h}, y_{h, t r i c .}=\frac{(3-u)(5-u)}{4(2-u)}
$$

where

$$
u=\frac{2}{\pi} \arccos \left(\frac{\sqrt{q}}{2}\right)
$$

so that $0 \leq u \leq 1$ for $4 \geq q \geq 0$. The angle $\theta$ in (2.25) is equal to $\pi u$ in (2.29), and hence

$$
q=q_{d, k} \Leftrightarrow u=\frac{2 k}{2 d+1}
$$

Note that the converse does not hold; that is, there are rational values of $u$ that are not of the form (2.30) and hence do not correspond to any of the $q_{d, k}$. An example is $u=1 / 2$.

\section{Determination of $n_{P}\left(L_{y}, d\right)$ for Cyclic Strips of the Square and Triangular Lattices}

In this section and the next we use (2.1) together with two theorems (eqs. (3.10 and (4.1) below) to determine structural properties of the chromatic polynomial and the full Potts model partition function for cyclic strip graphs $G$ of the square and triangular lattices. Let us define $n_{P}\left(L_{y}, d\right)$ as the number of terms $\lambda_{P, G, j}$ in $P(G, q)$ that have as their coefficients

$c_{P, G, j}=c^{(d)}$ and $n_{Z}\left(L_{y}, d\right)$ as the number of terms $\lambda_{Z, G, j}$ in $Z(G, q, v)$ that have as their coefficients $c_{Z, G, j}=c^{(d)}$. For the cyclic strip graphs under consideration here, these coefficients are independent of $L_{x}$ and depend on $L_{y}$ and $d$; furthermore, they are the same for both square and triangular strips, as discussed further below. While the individual $\lambda_{P, G, j}$ 's in (1.10) are, in general, different for the cyclic strips of the square and triangular lattices, the total number of $\lambda_{P, G, j}$ 's is the same, so we use the short notation $N_{P, L_{y}, \lambda}$. The same is true for the full partition function, so we use the notation $N_{Z, L_{y}, \lambda}$.

Since the chromatic polynomial $P(G, q)$ is the special case $v=-1$ (i.e. zero-temperature antiferromagnet) of the general (finite-temperature, $J$ positive or negative) Potts model partition function, (1.6), it follows that

$$
n_{P}\left(L_{y}, d\right) \leq n_{Z}\left(L_{y}, d\right)
$$

The total number, $N_{P, G, \lambda}$, of different terms $\lambda_{P, G, j}$ in (1.10) is given by

$$
N_{P, L_{y}, \lambda}=\sum_{d=0}^{L_{y}} n_{P}\left(L_{y}, d\right) .
$$

Since each term $\lambda_{P, G, j}$ is a distinct eigenvalue of the coloring matrix $\mathcal{T}$, the number $N_{P, L_{y}, \lambda}$ is the number of different invariant subspaces in the full $\mathcal{N}$-dimensional space of coloring configurations of the transverse slices of the strips. Similarly, for the full Potts model partition 
function,

$$
N_{Z, L_{y}, \lambda}=\sum_{d=0}^{L_{y}} n_{Z}\left(L_{y}, d\right) .
$$

and analogously this represents the number of different invariant subspaces for the matrix $\mathcal{T}_{Z}$. It was shown in [44] that the $\lambda_{P, G\left(L_{y}\right), j}$ 's are the same for the cyclic and Möbius strips (although the corresponding $c_{P, G, j}$ 's are different)

$$
\lambda_{P, G\left(L_{y}\right), F B C_{y}, P B C_{x}, j}=\lambda_{P, G\left(L_{y}\right), F B C_{y}, T P B C_{x}, j} \quad \forall j
$$

and hence the total number of terms is also the same:

$$
N_{P, G\left(L_{y}\right), F B C_{y}, P B C_{x}, \lambda}=N_{P, G\left(L_{y}\right), F B C_{y}, T P B C_{x}, \lambda} .
$$

The argument in [44] relied upon the local nature of the deletion-contraction operations used in calculating $P(G, q)$, and the same property is true of the deletion-contraction theorem used for calculating the Tutte polynomial, or equivalently, the Potts model partition function, so that the following generalizations of $(3.5)$ holds $[15,16]$ :

$$
\lambda_{Z, G\left(L_{y}\right), F B C_{y}, P B C_{x}, j}=\lambda_{Z, G\left(L_{y}\right), F B C_{y}, T P B C_{x}, j} \quad \forall j
$$

and hence

$$
N_{Z, G\left(L_{y}\right), F B C_{y}, P B C_{x}, \lambda}=N_{Z, G\left(L_{y}\right), F B C_{y}, T P B C_{x}, \lambda} .
$$

For the sum of the coefficients in (1.10), i.e., (1.16), we have

$$
C_{P, L_{y}}=\sum_{j=1}^{N_{P, L_{y}, \lambda}} c_{P, L_{y}, j}=\sum_{d=0}^{L_{y}} n_{P}\left(L_{y}, d\right) c^{(d)}
$$

and for the corresponding sum of coefficients in (1.9),

$$
C_{Z, L_{y}}=\sum_{j=1}^{N_{Z, L_{y}, \lambda}} c_{Z, L_{y}, j}=\sum_{d=0}^{L_{y}} n_{Z}\left(L_{y}, d\right) c^{(d)} .
$$

We first recall a theorem specifying $C_{P, L_{y}}$ for cyclic strips of the square and triangular lattice [44]:

Theorem 1.

$$
C_{P, L_{y}}=P\left(T_{L_{y}}, q\right)=q(q-1)^{L_{y}-1}
$$

Proof. Using coloring matrix methods $[22,43]$, one has that $C_{P, L_{y}}$ is equal to the chromatic polynomial for the coloring of the transverse slice of the strip, which is a line with $L_{y}$ vertices. This is a special case of a tree graph, for which the elementary general result in eq. (1.13) holds. 
Next, we have

Theorem 2. The $n_{P}\left(L_{y}, d\right), d=0,1, . . L_{y}$ are determined as follows. One has

$$
\begin{gathered}
n_{P}\left(L_{y}, d\right)=0 \text { for } d>L_{y}, \\
n_{P}\left(L_{y}, L_{y}\right)=1
\end{gathered}
$$

and

$$
n_{P}(1,0)=1
$$

with all other numbers $n_{P}\left(L_{y}, d\right)$ being determined by the two recursion relations

$$
n_{P}\left(L_{y}+1,0\right)=n_{P}\left(L_{y}, 1\right)
$$

and

$n_{P}\left(L_{y}+1, d\right)=n_{P}\left(L_{y}, d-1\right)+n_{P}\left(L_{y}, d\right)+n_{P}\left(L_{y}, d+1\right) \quad$ for $\quad L_{y} \geq 1 \quad$ and $\quad 1 \leq d \leq L_{y}+1$.

Proof. We substitute for $c^{(d)}$ from eq. (2.1) in eq. (3.10). We obtain another equation by differentiating this with respect to $q$ once; another by differentiating twice, and so forth up to $L_{y}$-fold differentiations. This yields $L_{y}+1$ linear equations in the $L_{y}+1$ unknowns, $n_{P}\left(L_{y}, d\right), d=0,1, \ldots, L_{y}$. We solve this set of equations to get the $n_{P}\left(L_{y}, d\right)$.

A corollary is that

$$
n_{P}\left(L_{y}, L_{y}-1\right)=L_{y} .
$$

The numbers $n_{P}\left(L_{y}, d\right)$ can be viewed as integer sequences in $L_{y}$ for a given value of $d$. We find that (for $L_{y} \geq 1$ where our strips are defined) $n_{P}\left(L_{y}, 0\right)$ is a Motzkin number [63]-[69]

$$
n_{P}\left(L_{y}, 0\right)=M_{L_{y}-1}
$$

where the Motzkin number $M_{n}$ is given by

$$
M_{n}=\sum_{j=0}^{n}(-1)^{j} C_{n+1-j}\left(\begin{array}{l}
n \\
j
\end{array}\right)
$$

where

$$
C_{n}=\frac{1}{n+1}\left(\begin{array}{c}
2 n \\
n
\end{array}\right)
$$

is the Catalan number. (We also use the symbol $C_{n}$ to denote the circuit graph with $n$ vertices; the meaning will be clear from context.) The Catalan and Motzkin numbers occur in many combinatoric applications [63]-[69]. Among these is the construction of nonintersecting chords on a circle; the number of ways of connecting a subset of $n$ points on a 
Table 1: Table of numbers $n_{P}\left(L_{y}, d\right)$ and their sums, $N_{P, L_{y}, \lambda}$ for cyclic strips of the square and triangular lattices. Blank entries are zero.

\begin{tabular}{|c|c|c|c|c|c|c|c|c|c|c|c|c|}
\hline \hline$L_{y} \downarrow d \rightarrow$ & 0 & 1 & 2 & 3 & 4 & 5 & 6 & 7 & 8 & 9 & 10 & $N_{P, L_{y}, \lambda}$ \\
\hline \hline 1 & 1 & 1 & & & & & & & & & & 2 \\
\hline 2 & 1 & 2 & 1 & & & & & & & & & 4 \\
\hline 3 & 2 & 4 & 3 & 1 & & & & & & & & 10 \\
\hline 4 & 4 & 9 & 8 & 4 & 1 & & & & & & & 26 \\
\hline 5 & 9 & 21 & 21 & 13 & 5 & 1 & & & & & & 70 \\
\hline 6 & 21 & 51 & 55 & 39 & 19 & 6 & 1 & & & & & 192 \\
\hline 7 & 51 & 127 & 145 & 113 & 64 & 26 & 7 & 1 & & & & 534 \\
\hline 8 & 127 & 323 & 385 & 322 & 203 & 97 & 34 & 8 & 1 & & & 1500 \\
\hline 9 & 323 & 835 & 1030 & 910 & 622 & 334 & 139 & 43 & 9 & 1 & & 4246 \\
\hline 10 & 835 & 2188 & 2775 & 2562 & 1866 & 1095 & 516 & 191 & 53 & 10 & 1 & 12092 \\
\hline \hline
\end{tabular}

circle by non-intersecting chords is $M_{n}$, while the number of ways of completely connecting $2 n$ points on the circle by such chords is $C_{n}$. Summing over subsets of points connected by chords, this yields the well-known relation

$$
M_{n}=\sum_{k=0}^{\left[\frac{n}{2}\right]}\left(\begin{array}{c}
n \\
2 k
\end{array}\right) C_{k} .
$$

As a consequence of our relation (3.14), the equation (3.17) also implies that

$$
n_{P}\left(L_{y}, 1\right)=M_{L_{y}} .
$$

A generating function is

$$
G_{n_{P}\left(L_{y}, 0\right)}(x)=\frac{1-x-\sqrt{1-2 x-3 x^{2}}}{2 x^{2}}=\sum_{L_{y}=1}^{\infty} n_{P}\left(L_{y}, 0\right) x^{L_{y}-1} .
$$

In eq. (5.1.3) below we shall give an exact determination of the total number, $N_{P, L_{y}, \lambda}$, of types of terms $\lambda_{P, L_{y}, j}$. In Table 1 we list the numbers $n_{P}\left(L_{y}, d\right)$ and the sums $N_{P, L_{y}, \lambda}$ for the first several widths of cyclic strips of the square and triangular lattice, $1 \leq L_{y} \leq 10$. Others can easily be calculated using our general formulas.

Now let us consider a random walk on the nonnegative integers such that in each step the walker moves by $+1,-1$, or 0 units. Denoting $m(n, k)$ as the number of walks of length $n$ steps starting at 0 and ending at $k$, we obtain the Motzkin triangle in Table 2 . The first column, corresponding to $k=0$ is the number of walks defined above that return to the origin after $n$ steps. This is given by the Motzkin number defined in (3.18); $m(n, 0)=M_{n}$. 
Table 2: Table of numbers $m(n, k)$ for the number of a random walk from 0 to $k$ in $n$ steps and the row sums $\mathcal{S}_{n}$. Blank entries are zero.

\begin{tabular}{|c|c|c|c|c|c|c|c|c|c|c|c|}
\hline \hline$n \downarrow k \rightarrow$ & 0 & 1 & 2 & 3 & 4 & 5 & 6 & 7 & 8 & 9 & $\mathcal{S}_{n}$ \\
\hline \hline 0 & 1 & & & & & & & & & & 1 \\
\hline 1 & 1 & 1 & & & & & & & & & 2 \\
\hline 2 & 2 & 2 & 1 & & & & & & & & 5 \\
\hline 3 & 4 & 5 & 3 & 1 & & & & & & & 13 \\
\hline 4 & 9 & 12 & 9 & 4 & 1 & & & & & & 35 \\
\hline 5 & 21 & 30 & 25 & 14 & 5 & 1 & & & & & 96 \\
\hline 6 & 51 & 76 & 69 & 44 & 20 & 6 & 1 & & & & 267 \\
\hline 7 & 127 & 196 & 189 & 133 & 70 & 27 & 7 & 1 & & & 750 \\
\hline 8 & 323 & 512 & 518 & 392 & 230 & 104 & 35 & 8 & 1 & & 2123 \\
\hline 9 & 835 & 1353 & 1422 & 1140 & 726 & 369 & 147 & 44 & 9 & 1 & 4246 \\
\hline \hline
\end{tabular}

The second column, $m(n, 1)$, is given by the first differences of Motzkin numbers:

$$
m(n, 1)=\sum_{j=0}^{\left[\frac{n+1}{2}\right]} \frac{1}{j+1}\left(\begin{array}{c}
n \\
2 j-1
\end{array}\right)\left(\begin{array}{c}
2 j \\
j
\end{array}\right) .
$$

The row sums in Table 2 will be important below; we denote them as

$$
\mathcal{S}_{n}=\sum_{k=0}^{n} m(n, k)
$$

Notice that Table 1 can be viewed as the combination of two Motzkin triangles, i.e. Table 2 , as follows,

$$
n_{P}\left(L_{y}, d\right)=m\left(L_{y}-1, d\right)+m\left(L_{y}-1, d-1\right) .
$$

In light of the property that the coefficients (= multiplicities of eigenvalues of the transfer matrix $) c^{(d)}$ are Chebyshev polynomials of the second kind for the cyclic strips of the square and triangular lattices and for Möbius strips of the square lattice (discussed below), and the finding in eq. (3.17) for the numbers of $\lambda_{P, G, j}$ 's with coefficients $c^{(0)}$ and $c^{(1)}$, it is interesting to note that in [65] a connection has been shown between what is called a Motzkin polynomial and this Chebyshev polynomial. To see this, one defines $s_{h, 0}=M_{h}, s_{h, 1}=M_{h}-M_{h-1}$ and shows that, with $s_{h, n}=s_{h, n-1}-s_{h-1, n-1}-s_{h-2, n-2}$ for $1 \leq n \leq h$, it is possible to express $s_{h, n}$ as $s_{h, n}=a_{n} M_{h}+a_{n-1} M_{h-1}+\ldots+a_{0} M_{h-n}$, where the coefficients $a_{j}$ are independent of $h$. The Motzkin polynomial is defined as $S_{n}(x)=a_{n} x^{n}+\ldots+a_{1} x+a_{0}$, and the connection is that $S_{n}(x)=U_{n}\left(\frac{x-1}{2}\right)[65]$.

Certain ( $q$-independent) relations between the $n_{P}\left(L_{y}, d\right)$ can be derived by evaluating the sum (3.8) and its result (3.10) for $q=0,1$, and 2. Setting $q=0$ in (3.8) and (3.10), and 
using (2.17), we have

$$
\sum_{d=0}^{L_{y}} n_{P}\left(L_{y}, d\right) c^{(d)}(q=0)=\sum_{d=0}^{L_{y}}(-1)^{d} n_{P}\left(L_{y}, d\right)=0 .
$$

Next we evaluate (3.8) and (3.10) for $q=1$. If $L_{y}=1$, this only involves one term, $n_{P}(1,0)=1$. So consider $L_{y} \geq 2$ and set $L_{y}=3 k+r$ with integer $k \geq 0$ and $r=0$, 1 , or 2. To express the equation compactly, it is convenient to use a Heaviside step function $\theta(z)$, where $\theta(z)=1$ if $z>0$ and $\theta(z)=0$ if $z \leq 0$. Using (2.18), we have

$$
\begin{aligned}
\sum_{d=0}^{L_{y}} n_{P}\left(L_{y}, d\right) c^{(d)}(q=1) & =\sum_{j=0}^{k} n_{P}\left(L_{y}, 3 j\right)-\sum_{j=0}^{k-1+\theta\left(r-\frac{3}{2}\right)} n_{P}\left(L_{y}, 3 j+2\right) \\
& =0
\end{aligned}
$$

where here and in other summations, it is understood that if the upper limit on the summation is negative, the sum is zero. A similar relation can be obtained in a straightforward manner by substituting $q=2$ in (3.8) and (3.10). Other similar relations among the $n_{P}\left(L_{y}, d\right)$ can be obtained by evaluating (3.8) and (3.10) for $q=3$ and $q=4$; for these relations, the

right-hand sides grow with $L_{y}$, in contrast to (3.26)-(3.27), where the right-hand sides are constant.

\section{Determination of $n_{Z}\left(L_{y}, d\right)$ for Cyclic Strips of the Square and Triangular Lattices}

We next generalize these results to the full Potts model partition function. We determine the numbers $n_{Z}\left(L_{y}, d\right)$ by observing that in the cyclic strips under consideration here, the only difference between the coloring for the $T=0$ Potts antiferromagnet and for the general Potts model at finite temperature is the relaxation of the constraint that no two adjacent vertices can have the same color (indeed, for ferromagnetic coupling, there is a preference for these vertices to have the same color). Hence, one simply counts all possible colorings, and obtains

Theorem 3.

$$
\sum_{d=0}^{L_{y}} n_{Z}\left(L_{y}, d\right) c^{(d)}=q^{L_{y}}
$$

Next, we have

Theorem 4. The $n_{Z}\left(L_{y}, d\right)$ are determined as follows. One has

$$
n_{Z}\left(L_{y}, d\right)=0 \quad \text { for } \quad d>L_{y}
$$




$$
n_{Z}\left(L_{y}, L_{y}\right)=1
$$

and

$$
n_{Z}(1,0)=1 \text {. }
$$

All other numbers $n_{Z}\left(L_{y}, d\right)$ are then determined by the two recursion relations

$$
n_{Z}\left(L_{y}+1,0\right)=n_{Z}\left(L_{y}, 0\right)+n_{Z}\left(L_{y}, 1\right)
$$

and

$$
\begin{gathered}
n_{Z}\left(L_{y}+1, d\right)=n_{Z}\left(L_{y}, d-1\right)+2 n_{Z}\left(L_{y}, d\right)+n_{Z}\left(L_{y}, d+1\right) \\
\text { for } 1 \leq d \leq L_{y}+1 .
\end{gathered}
$$

Proof. The proof is similar to that for Theorem 2: we substitute for $c^{(d)}$ from eq. (2.1) in eq. (4.1). We obtain another equation by differentiating this with respect to $q$ once; another by differentiating twice, and so forth up to $L_{y}$-fold differentiations. This yields $L_{y}+1$ linear equations in the $L_{y}+1$ unknowns, $n_{Z}\left(L_{y}, d\right), d=0,1, \ldots, L_{y}$. We solve this set of equations to get the $n_{Z}\left(L_{y}, d\right)$.

A corollary is that

$$
n_{Z}\left(L_{y}, L_{y}-1\right)=2 L_{y}-1
$$

From Theorems 3 and 4 we find a general formula for the numbers $n_{Z}\left(L_{y}, d\right)$ :

$$
n_{Z}\left(L_{y}, d\right)=\frac{(2 d+1)}{\left(L_{y}+d+1\right)}\left(\begin{array}{c}
2 L_{y} \\
L_{y}-d
\end{array}\right)
$$

for $0 \leq d \leq L_{y}$, with $n_{Z}\left(L_{y}, d\right)=0$ for $d>L_{y}$. For fixed $d$ in the range $0 \leq d \leq L_{y}$ where $n_{Z}\left(L_{y}, d\right)$ is nonvanishing, it has the leading asymptotic behavior

$$
n_{Z}\left(L_{y}, d\right) \sim(2 d+1) \pi^{-1 / 2} L_{y}^{-3 / 2} 4^{L_{y}}\left[1+O\left(L_{y}^{-1}\right)\right] \quad \text { as } \quad L_{y} \rightarrow \infty \text { for fixed } d .
$$

(The formal notation $1+O\left(L_{y}^{-1}\right)$ in (4.9) and in other asymptotic expansions is not intended to indicate the sign of the coefficient of the $O\left(L_{y}^{-1}\right)$ term; here it is actually negative.) As a measure of the asymptotic behavior of $n_{Z}\left(L_{y}, d\right)$ when $d$, rather than being fixed, is a finite fraction of $L_{y}$, we take the central value $d=L_{y} / 2$ and calculate that

$$
n_{Z}\left(L_{y}, \frac{L_{y}}{2}\right) \sim \pi^{-1 / 2} L_{y}^{-1 / 2} 4^{2 L_{y}+1} 3^{-\frac{3}{2}\left(L_{y}+1\right)}\left[1+O\left(L_{y}^{-1}\right)\right] \quad \text { as } \quad L_{y} \rightarrow \infty .
$$

Note that for the special case $d=0$, this reduces to

$$
n_{Z}\left(L_{y}, 0\right)=C_{L_{y}}
$$


Table 3: Table of numbers $n_{Z}\left(L_{y}, d\right)$ and their sums, $N_{Z, G, \lambda}$. See text for general formulas.

\begin{tabular}{|c|c|c|c|c|c|c|c|c|c|c|c|c|}
\hline \hline$L_{y} \downarrow d \rightarrow$ & 0 & 1 & 2 & 3 & 4 & 5 & 6 & 7 & 8 & 9 & 10 & $N_{Z, L_{y}, \lambda}$ \\
\hline \hline 1 & 1 & 1 & & & & & & & & & & 2 \\
\hline 2 & 2 & 3 & 1 & & & & & & & & & 6 \\
\hline 3 & 5 & 9 & 5 & 1 & & & & & & & & 20 \\
\hline 4 & 14 & 28 & 20 & 7 & 1 & & & & & & & 70 \\
\hline 5 & 42 & 90 & 75 & 35 & 9 & 1 & & & & & & 252 \\
\hline 6 & 132 & 297 & 275 & 154 & 54 & 11 & 1 & & & & & 924 \\
\hline 7 & 429 & 1001 & 1001 & 637 & 273 & 77 & 13 & 1 & & & & 3432 \\
\hline 8 & 1430 & 3432 & 3640 & 2548 & 1260 & 440 & 104 & 15 & 1 & & & 12870 \\
\hline 9 & 4862 & 11934 & 13260 & 9996 & 5508 & 2244 & 663 & 135 & 17 & 1 & & 48620 \\
\hline 10 & 16796 & 41990 & 48450 & 38760 & 23256 & 10659 & 3705 & 950 & 170 & 19 & 1 & 184756 \\
\hline \hline
\end{tabular}

where the Catalan number $C_{n}$ was defined in (3.19). In Table 3 we list the first few numbers $n_{Z}\left(L_{y}, d\right)$ and the total sums $N_{Z, L_{y}, \lambda}$ that will be calculated below in (5.2.2).

Similar to Table 2, we can also make up a Catalan triangle, and shall find that Table 3 is just the combination of two triangles.

Certain ( $q$-independent) relations between the $n_{Z}\left(L_{y}, d\right)$ can be derived by evaluating the sum (3.9) and its result (4.1) for $q=0$ and $q=1$. Setting $q=0$ in this sums and using (2.17), we have

$$
\sum_{d=0}^{L_{y}}(-1)^{d} n_{Z}\left(L_{y}, d\right)=0 .
$$

Next we evaluate (3.9) and (4.1) for $q=1$. Let $L_{y}=3 k+r$ with $k \geq 0$ and $r=0,1$, or 2 . Using (2.18), we have

$$
\sum_{j=0}^{k} n_{Z}\left(L_{y}, 3 j\right)-\sum_{j=0}^{k-1+\theta\left(r-\frac{3}{2}\right)} n_{Z}\left(L_{y}, 3 j+2\right)=1 .
$$

We have obtained the following relations involving both $n_{Z}\left(L_{y}, d\right)$ and $n_{P}\left(L_{y}, d\right)$, as well as the total numbers $N_{Z, L_{y}, \lambda}$ and $N_{P, L_{y}, \lambda}$

$$
n_{P}\left(L_{y}, d\right)=\sum_{j=0}^{L_{y}-1}(-1)^{j}\left(\begin{array}{c}
L_{y}-1 \\
j
\end{array}\right) n_{Z}\left(L_{y}-j, d\right)
$$


and

$$
n_{Z}\left(L_{y}, d\right)=\sum_{j=0}^{L_{y}-1}\left(\begin{array}{c}
L_{y}-1 \\
j
\end{array}\right) n_{P}\left(L_{y}-j, d\right) .
$$

Since the total numbers of terms $N_{P, L_{y}, \lambda}$ and $N_{Z, L_{y}, \lambda}$ are sums of the $n_{P}\left(L_{y}, d\right)$ and $n_{Z}\left(L_{y}, d\right)$ given, respectively, by (3.2) and (3.3), it follows that

$$
N_{P, L_{y}, \lambda}=\sum_{j=0}^{L_{y}-1}(-1)^{j}\left(\begin{array}{c}
L_{y}-1 \\
j
\end{array}\right) N_{Z, L_{y}-j, \lambda}
$$

and

$$
N_{Z, L_{y}, \lambda}=\sum_{j=0}^{L_{y}-1}\left(\begin{array}{c}
L_{y}-1 \\
j
\end{array}\right) N_{P, L_{y}-j, \lambda}
$$

Note that for $d=0$, our eq. (4.14) reduces to the relation (3.18) expressing the Motzkin number as a certain weighted sum of Catalan numbers, while eq. (4.15) reduces to the relation expressing the Catalan number as a weighted sum of Motzkin numbers (e.g., [67])

$$
C_{n}=\sum_{j=0}^{n-1}\left(\begin{array}{c}
n-1 \\
j
\end{array}\right) M_{n-j-1}=\sum_{k=0}^{n-1}\left(\begin{array}{c}
n-1 \\
k
\end{array}\right) M_{k}
$$

for $n \geq 1$ (with $C_{0}=1$ ). Further, for the even and odd widths $L_{y}=2 \ell$ and $L_{y}=2 \ell+1$,

$$
N_{Z, \ell, \lambda}=\sum_{j=0}^{2 \ell-1}(-1)^{j}\left(\begin{array}{c}
2 \ell-1 \\
j
\end{array}\right) N_{P, 2 \ell-j, \lambda}
$$

and

$$
2 N_{Z, \ell, \lambda}=\sum_{j=0}^{2 \ell}(-1)^{j}\left(\begin{array}{c}
2 \ell \\
j
\end{array}\right) N_{P, 2 \ell+1-j, \lambda} .
$$

\section{Determination of $N_{P, L_{y}, \lambda}$ and $N_{Z, L_{y}, \lambda}$ for Cyclic and Möbius Strips of the Square and Triangular Lattices and Relation with Directed Lattice Animals}

In this section we shall use our results above to calculate the total number, $N_{P, G, \lambda}$, of $\lambda_{P, G, j}$ 's in the chromatic polynomial (1.10) and the total number, $N_{Z, G, \lambda}$, of $\lambda_{Z, G, j}$ 's in the full Potts model partition function for cyclic strips $G$ of the square and triangular lattice. Since the individual numbers $n_{P}\left(L_{y}, d\right)$ were shown to be the same for the cyclic strips of the square and triangular lattices, and similarly for $n_{Z}\left(L_{y}, d\right)$, clearly it is also true that the respective total 
numbers $N_{P, G, \lambda}$ and $N_{Z, G, \lambda}$ are the same for cyclic strips of the square and triangular lattices. Furthermore, as a consequence of eqs. (3.4)-(3.7), our use of cyclic strips to calculate the total numbers $N_{P, G, \lambda}$ and $N_{Z, G, \lambda}$ also yields these respective numbers for the Möbius strips of the square and triangular lattices. This is useful since, as will be seen in the next sections, the individual coefficients involve a larger set for Möbius strips of the square lattice, namely not just $c^{(d)}$ but also $-c^{(d)}$; moreover, the coefficients for Möbius strips of the triangular lattice are not, in general, polynomials in $q[42]$.

\section{$5.1 \quad N_{P, L_{y}, \lambda}$}

For the total number of terms $N_{P, L_{y}, \lambda}$, from our theorems above we obtain the recursion relation

$$
N_{P, L_{y}+1, \lambda}=3 N_{P, L_{y}, \lambda}-2 n_{P}\left(L_{y}, 0\right) .
$$

We find that

$$
N_{P, L_{y}, \lambda}=2 \mathcal{S}_{L_{y}}=2\left(L_{y}-1\right) ! \sum_{j=0}^{\left[\frac{L_{y}}{2}\right]} \frac{\left(L_{y}-j\right)}{(j !)^{2}\left(L_{y}-2 j\right) !}
$$

where $\mathcal{S}_{n}$ was given in (3.24). A generating function for these numbers is

$$
G_{N_{P, L_{y}, \lambda}}(x)=\left(\frac{1+x}{1-3 x}\right)^{1 / 2}-1=\sum_{L_{y}=1}^{\infty} N_{P, L_{y}, \lambda} x^{L_{y}} .
$$

From this, it follows that the number $N_{P, L_{y}, \lambda}$ grows exponentially fast with the width $L_{y}$ of the cyclic or Möbius strip of the square or triangular lattice, with the leading asymptotic behavior

$$
N_{P, L_{y}, \lambda} \sim L_{y}^{-1 / 2} 3^{L_{y}} \quad \text { as } \quad L_{y} \rightarrow \infty
$$

\section{$5.2 \quad N_{Z, G, \lambda}$}

A corollary of Theorem 4 is that

$$
N_{Z, L_{y}+1, \lambda}=4 N_{Z, L_{y}, \lambda}-2 n_{Z}\left(L_{y}, 0\right) .
$$

Summing the individual numbers $n_{Z}\left(L_{y}, d\right)$ to evaluate the total, (3.3), we have, for cyclic and Möbius strips of width $L_{y}$ of the square and triangular lattices,

$$
\begin{aligned}
N_{Z, L_{y}, \lambda} & =\sum_{d=0}^{L_{y}} \frac{(2 d+1)}{\left(L_{y}+d+1\right)}\left(\begin{array}{c}
2 L_{y} \\
L_{y}-d
\end{array}\right) \\
& =\left(\begin{array}{c}
2 L_{y} \\
L_{y}
\end{array}\right) .
\end{aligned}
$$

As $L_{y} \rightarrow \infty, N_{Z, L_{y}, \lambda}$ has the leading asymptotic behavior

$$
N_{Z, L_{y}, \lambda} \sim \pi^{-1 / 2} L_{y}^{-1 / 2} 4^{L_{y}}\left[1+O\left(L_{y}^{-1}\right)\right] \quad \text { as } L_{y} \rightarrow \infty .
$$


We give two comparisons of the asymptotic behavior of individual numbers $n_{Z}\left(L_{y}, d\right)$ with the total, $N_{Z, L_{y}, \lambda}$. First, we compare the growth of $n_{Z}\left(L_{y}, d\right)$ for fixed $d$ (in the range $0 \leq d \leq L_{y}$ where $n_{Z}\left(L_{y}, d\right)$ is nonzero) with the growth of $N_{Z, L_{y}, \lambda}$ :

$$
\frac{n_{Z}\left(L_{y}, d\right)}{N_{Z, L_{y}, \lambda}} \sim \frac{(2 d+1)}{L_{y}}\left[1+O\left(L_{y}^{-1}\right)\right] \quad \text { as } \quad L_{y} \rightarrow \infty \text { for fixed } d .
$$

Second, we compare the relative growths of the central number $n_{Z}\left(L_{y}, \frac{L_{y}}{2}\right)$ and the total, $N_{Z, L_{y}, \lambda}$ :

$$
\begin{aligned}
\frac{n_{Z}\left(L_{y}, \frac{L_{y}}{2}\right)}{N_{Z, L_{y}, \lambda}} \sim\left(\frac{4}{3^{3 / 2}}\right)^{L_{y}+1}\left[1+O\left(L_{y}^{-1}\right)\right] & \sim(0.769800 \ldots)^{L_{y}+1}\left[1+O\left(L_{y}^{-1}\right)\right] \\
& \quad \text { as } L_{y} \rightarrow \infty .
\end{aligned}
$$

\subsection{Connection with Directed Lattice Animals}

For an arbitrary $G$, the Potts model partition function $Z(G, q, v)$ gives information about certain graph-theoretic quantities describing $G$, as is clear from the Kasteleyn-Fortuin representation (1.5) and the equivalence in eqs. (12.1.6) and (12.1.9) to the Tutte polynomial and Whitney rank polynomial. An example of this information is illustrated by the zerotemperature antiferromagnetic special case $P(G, q)=Z(G, q,-1)$ in eq. (1.6), i.e., the chromatic polynomial, counting the number of proper vertex colorings of $G$. Other examples are that the Tutte polynomials $T(G, x, y)$ for various special values of its arguments, counts the number of spanning trees, spanning forests, connected spanning subgraphs, and spanning subgraphs of $G$ (see appendix). It is also well known that the $q \rightarrow 1$ limit of the Potts model is related to bond percolation and the $q \rightarrow 1$ limit of a certain multisite Potts model is related to site percolation, as reviewed in [2].

Here we would like to report two new and very interesting relations with graph-theoretic quantities that we have found from our calculations. These involve directed lattice animals. To explain this, we recall that an animal $A$ on a lattice or more generally a graph $G$ is defined as a (finite) set of vertices in $G$ with the property that any two vertices in $A$ are connected by means of a path in $G$ having all its vertices in $A$. Thus, any vertex in $A$ is adjacent to another vertex in $A$. If the graph is a lattice, the animal is termed a lattice animal, and on an infinite lattice, the lattice animals are defined up to an overall lattice translation. A directed lattice animal $A_{d i r}$ is an animal on a lattice $\Lambda$ together with a special origin or root point $O$ such that any vertex of $A$ can be reached starting from $O$ by an oriented path on $\Lambda$ having all of its vertices in $A$. For example, a directed lattice animal on the square lattice could be defined by restricting the orientations of each step of the oriented path to be "eastward" or "northward", so that, starting at the origin, this animal would extend in the northeast quadrant and would not contain any backtracking steps going in the south or west 
directions. (The animals that we consider here are also called site animals to distinguish them from bond animals; we shall leave this implicit.) Animals and directed animals are related, respectively, to percolation and directed percolation. Let us denote $N_{A, \Lambda, n}$ and $N_{D A, \Lambda, n}$ as the total number of lattice animals and directed lattice animals with $n$ vertices, respectively, on the lattice $\Lambda$. For $n \rightarrow \infty$, the numbers of lattice animals and directed lattice animals grow asymptotically like $n^{-\theta} a^{n}$, where $a$ depends on the lattice and, e.g., for 2D lattices, $\theta=1 / 2$ (e.g. [70]-[76] and references therein). Having given this background, we now state the relations that we have obtained.

First, we find that the total number of distinct eigenvalues of the coloring matrix, i.e. the total number, $N_{P, L_{y}, \lambda}$, of different $\lambda_{P, G, j}$ 's in the chromatic polynomial (1.10) for cyclic strips with width $L_{y}$ of the square and triangular lattice (equal by (3.5) to the same number for the corresponding Möbius strips of the square and triangular lattices) is twice the number of directed lattice animals with $n=L_{y}$ vertices on the square (sq) lattice:

$$
N_{P, L_{y}, \lambda}=2 N_{D A, s q, L_{y}} .
$$

We established this by observing that the generating function for directed lattice animals on the square lattice, which is known exactly [70,71], is precisely $(1 / 2)$ times the generating function (5.1.3).

Second, we find that the analogous total number, $N_{Z, L_{y}, \lambda}$, of different eigenvalues $\lambda_{Z, G, j}$ 's appearing in the full Potts model partition function (1.9) for these cyclic and Möbius strips with width $L_{y}$ of the square and triangular lattices is twice the number of directed lattice animals with $n=L_{y}$ vertices on the triangular lattice:

$$
N_{Z, L_{y}, \lambda}=2 N_{D A, t r i, L_{y}} .
$$

We established this by recalling the known result $[70,71]$

$$
N_{D A, t r i, n}=\frac{1}{2}\left(\begin{array}{c}
2 n \\
n
\end{array}\right)
$$

and using our calculation (5.2.2). It should be emphasized that, as we have shown above, the numbers $N_{P, L_{y}, \lambda}$ and $N_{Z, L_{y}, \lambda}$ on the respective left-hand sides of (5.3.1) and (5.3.2) apply for cyclic and Möbius strips of both the square and triangular lattice, whereas the right-hand side of (5.3.1) is specific to the square lattice and the right-hand side of (5.3.2) is specific to the triangular lattice.

Parenthetically, we mention some other relations. The enumeration of directed lattice animals on the square and triangular lattices was shown in [71] to be connected with the hard-square lattice gas model [72]. As discussed in [75, 76], no exact result has been obtained, analogous to those in [70,71], for the number of directed lattice animals on the honeycomb lattice. Directed lattice animals are connected with directed percolation, and the latter has been related to a kind of chiral Potts model [77]. Compact lattice animals are the subset of lattice animals with no unoccupied interior sites; directed compact lattice animals have been related to the $q \rightarrow \infty$ limit of the Potts model and have been shown to have numbers that grow less rapidly than the leading $a^{n}$ growth in the numbers of regular directed lattice animals [78]. 


\section{Determination of $n_{P, M b}\left(L_{y}, d, \pm\right)$ for Möbius Strips of the Square Lattice}

Möbius strips differ in their global topology from cyclic strips. For the Möbius strips of the square lattice that have been studied, the coefficients are polynomials in $q$, but the polynomials that occur for the strip of width $L_{y}$ arise from the set $\pm c^{(d)}$, where $0 \leq d \leq L_{y} / 2$ for even $L_{y}$ and $0 \leq d \leq\left(L_{y}+1\right) / 2$ for odd $L_{y}$, rather than the set $c^{(d)}, 0 \leq d \leq L_{y}$ as in the case of the cyclic strips. In passing, we note that for Möbius strips of the triangular lattice, we have found, originally for width $L_{y}=2$ in [42] and later for width $L_{y}=3$ in [46], that in the case of chromatic polynomials, some of the coefficients are not polynomials, but rather, algebraic functions of $q[42,46]$; similarly, some of the coefficients in the full Potts model partition function for the Möbius strip of the triangular lattice are not polynomials in $q$ [47]. This is related to the fact, discussed before [46, 47], that the Möbius strip of the triangular lattice involves a seam, i.e., it is not translationally homogeneous in the longitudinal direction, whereas the Möbius strip of the square lattice does not have a seam and is translationally homogeneous in the longitudinal direction. Hence, for Möbius strips, we only consider the square lattice here.

Since both signs of the $c^{(d)}$ occur, it is necessary to define a larger set of numbers for Möbius strips of the square lattice. Let $n_{P, M b}\left(L_{y}, d, \pm\right)$ denote the number of terms $\lambda_{j}$ 's in the expression (1.10) for $P\left(s q, L_{y} \times L_{x}, F B C_{y}, T P B C_{x}, q\right)$ with coefficients $c_{P, L_{y}, M b, j}= \pm c^{(d)}$, respectively, where $M b$ denotes Möbius. The notation $c_{P, L_{y}, M b, j}$ reflects the fact that these coefficients depend on $L_{y}$ and the Möbius boundary conditions, but are independent of $L_{x}$. The sums and differences for each $d$ are defined as

$$
n_{P, M b, t o t}\left(L_{y}, d\right)=n_{P, M b}\left(L_{y}, d,+\right)+n_{P, M b}\left(L_{y}, d,-\right)
$$

and

$$
\Delta n_{P, M b}\left(L_{y}, d\right)=n_{P, M b}\left(L_{y}, d,+\right)-n_{P, M b}\left(L_{y}, d,-\right) .
$$

We first obtain a general theorem for the sum of the coefficients:

Theorem 5 .

$$
C_{P, L_{y}, M b}=\sum_{j=1}^{N_{P, L_{y}, \lambda}} c_{P, L_{y}, M b, j}=\sum_{d=0}^{d_{\max }} \Delta n_{P, M b}\left(L_{y}, d\right) c^{(d)}= \begin{cases}0 & \text { for even } L_{y} \\ P\left(T_{\left(\frac{L_{y}+1}{2}\right)}, q\right) & \text { for odd } L_{y}\end{cases}
$$

where $P\left(T_{n}, q\right)$ was given in (1.13) and

$$
d_{\text {max }}=\left\{\begin{array}{ll}
\frac{L_{y}}{2} & \text { for even } L_{y} \\
\frac{\left(L_{y}+1\right)}{2} & \text { for odd } L_{y}
\end{array} .\right.
$$

Proof. Our method for proving this theorem is inspired by coloring matrix methods [43]. The Möbius strip involves a reversed-orientation periodic longitudinal boundary condition. 
We can think of constructing such a strip by cutting a cyclic strip, reversing the orientation of one of the ends, and gluing these ends together again. For a strip with $L_{y}$ even, let us label the vertices on the two ends as $1,2, . ., L_{y}$; then the Möbius boundary condition means identifying vertex 1 with vertex $L_{y}$, vertex 2 with vertex $L_{y}-1$, and so forth. As regards the coloring constraint, in the case $L_{y}=2$, this effectively produces a subgraph consisting of a single vertex with an edge forming a loop. In the case $L_{y}=4$, this produces a vertex $1=4$ with two edges connecting to a vertex $2=3$, which in turn has a loop connected to it, and so forth for higher even values of $L_{y}$. Because of the loop that appears in each even- $L_{y}$ case, the chromatic polynomial for coloring this subgraph vanishes identically. (The value of $d_{\max }$ given in (6.4) follows from this graphical construction.) This proves the theorem for the case of even $L_{y}$. For odd $L_{y}$, consider first the case $L_{y}=3$; here the Möbius boundary condition identifies vertex 1 with vertex 3 and leaves vertex 2 invariant. Hence, as regards the coloring, it effectively produces a subgraph consisting of the vertex $1=3$ connected by two edges with the vertex 2 . The chromatic polynomial for the coloring of this subgraph is not sensitive to the multiple edges and hence is $P\left(T_{2}, q\right)=q(q-1)$. Again, this subgraph construction yields the value of $d_{\max }$ for odd $L_{y}$ in (6.4). In a similar manner, for higher odd values of $L_{y}$, the Möbius boundary condition leads to a subgraph with $\left(L_{y}+1\right) / 2$ vertices forming a chain, with each interior pair connected to the next by two edges. The chromatic polynomial for the coloring of this subgraph is $P\left(T_{\left(L_{y}+1\right) / 2}, q\right)=q(q-1)^{\left(L_{y}-1\right) / 2}$. This completes the proof of the theorem.

Two corollaries of this theorem are as follows.

Corollary 1

$$
n_{P, M b}\left(L_{y}, d,+\right)=n_{P, M b}\left(L_{y}, d,-\right) \quad \text { for even } \quad L_{y}
$$

Proof. If $L_{y}$ is even, then in order for the terms of the highest power in $q$ to cancel so as to yield a sum of 0 as in (6.3), it is necessary that $n_{P, M b}\left(L_{y}, d_{\max },+\right)=n_{P, M b}\left(L_{y}, d_{\max },-\right)$, where $d_{\max }$ was given above in (6.4). But then in turn, in order for the terms of degree $d_{\max }-1$ to sum to zero, it is necessary that $n_{P, M b}\left(L_{y}, d_{\max }-1,+\right)=n_{P, M b}\left(L_{y}, d_{\max }-1,-\right)$, and so forth for all powers.

Corollary 2

$$
\Delta n_{P, M b}\left(L_{y}, d\right)=n_{P}\left(\frac{L_{y}+1}{2}, d\right) \text { for odd } \quad L_{y} \geq 3
$$

Proof. This follows from eq. (6.3) by the same kind of argument that was used to obtain the $n_{P}\left(L_{y}, d\right)$. Given that the sum $C_{P, L_{y}, M b}$ satisfies (6.3), this uniquely determines the $\Delta n_{P, M b}\left(L_{y}, d\right)$ 's just as $(3.10)$ determined the $n_{P}\left(L_{y}, d\right)$. 
In order to determine the numbers of coefficients $c^{(d)}$, i.e., $n_{P, M b}\left(L_{y}, d, \pm\right)$, for a Möbius strip of width $L_{y}$, we start with the chromatic polynomial for the cyclic $L_{y}$ strip and determine how the coefficients $c^{(d)}$ change when one changes the longitudinal boundary condition from cyclic to Möbius. The next two theorems determines this (the proofs are given after the second of these two theorems).

Theorem 6 Consider an $L_{y} \times L_{x}$ strip of the square lattice with $\left(F B C_{y}, P B C_{x}\right)$. As before, the coefficients $c_{P, G, j}$ are made up from the set $c^{(d)}$ with $0 \leq d \leq L_{y}$. When one changes the longitudinal boundary condition from cyclic to Möbius, the following respective changes of coefficients of even degree $d=2 k$ and of odd degree $2 k+1$ occur:

$$
\begin{gathered}
c^{(0)} \rightarrow \pm c^{(0)} \\
c^{(2 k)} \rightarrow \pm c^{(k-1)}, 1 \leq k \leq\left[\frac{L_{y}}{2}\right] \\
c^{(2 k+1)} \rightarrow \pm c^{(k+1)}, 0 \leq k \leq\left[\frac{L_{y}-1}{2}\right]
\end{gathered}
$$

where in eqs. (6.8) and (6.9), we again use the notation $[\nu]$ to denote the integral part of $\nu$. Thus, if there are $n_{P}\left(L_{y}, 0\right)$ coefficients $c_{P, G, j}$ of the terms $\left(\lambda_{j}\right)^{m}$ of the form $c^{(0)}$ in eq. (1.10) for $P\left(s q\left(L_{y} \times L_{x} ; F B C_{y}, P B C_{x}\right), q\right)$, then the respective coefficients multiplying the terms $\left(\lambda_{j}\right)^{m}$ in the chromatic polynomial for the corresponding Möbius strip, $P\left(s q\left(L_{y} \times L_{x} ; F B C_{y}, T P B C_{x}\right), q\right)$, are either of the form $+c^{(0)}$ or $-c^{(0)}$, and so forth for the $c^{(d)}$ with $d>0$, as specified by eqs. (6.8) and (6.9).

Theorem 7. With the same premise as in Theorem 6 , we have

$$
\begin{aligned}
& n_{P, M b}\left(L_{y}, 0, \pm\right)=\frac{1}{2} n_{P, M b, t o t}\left(L_{y}, 0\right)=\frac{1}{2}\left[n_{P}\left(L_{y}, 0\right)+n_{P}\left(L_{y}, 2\right)\right] \quad \text { for } L_{y} \text { even } \\
& n_{P, M b}\left(L_{y}, d, \pm\right)=\frac{1}{2} n_{P, M b, t o t}\left(L_{y}, d\right)=\frac{1}{2}\left[n_{P}\left(L_{y}, 2 d-1\right)+n_{P}\left(L_{y}, 2 d+2\right)\right] \\
& \text { for } L_{y} \text { even and } 1 \leq d \leq \frac{L_{y}}{2} \\
& n_{P, M b}\left(L_{y}, d, \pm\right)=0 \text { for } L_{y} \text { even and } d \geq \frac{L_{y}+2}{2} \\
& n_{P, M b}\left(L_{y}, 0, \pm\right)=\frac{1}{2}\left[n_{P}\left(L_{y}, 0\right)+n_{P}\left(L_{y}, 2\right) \pm n_{P}\left(\frac{L_{y}+1}{2}, 0\right)\right] \text { for } \quad L_{y} \text { odd } \\
& n_{P, M b}\left(L_{y}, d, \pm\right)=\frac{1}{2}\left[n_{P}\left(L_{y}, 2 d-1\right)+n_{P}\left(L_{y}, 2 d+2\right) \pm n_{P}\left(\frac{L_{y}+1}{2}, d\right)\right]
\end{aligned}
$$




$$
\begin{array}{r}
\text { for } L_{y} \text { odd and } 1 \leq d \leq \frac{L_{y}+1}{2} \\
n_{P, M b}\left(L_{y}, d, \pm\right)=0 \quad \text { for } L_{y} \text { odd } \text { and } d \geq \frac{L_{y}+3}{2}
\end{array}
$$

Proofs. We prove these theorems using an inductive argument. From the chromatic polynomials for the cyclic and Möbius strips of the square lattice with width $L_{y}=2$ [18], one knows the transformation rule for the coefficients $c^{(d)}$ with $0 \leq d \leq 2$ as one goes from cyclic to Möbius longitudinal boundary conditions, namely, $c^{(0)} \rightarrow \pm c^{(0)}, c^{(1)} \rightarrow \pm c^{(1)}$, and $c^{(2)} \rightarrow \pm c^{(0)}$, where the sign information will not be needed. For $L_{y}=3$, we know the numbers $n_{P}\left(L_{y}, d\right)$ for the cyclic strip from the previous theorem. We also know the differences of the numbers of coefficients of each degree for the $L_{y}=3$ Möbius strip, $\Delta n_{P, M b}(3, d)$; these are determined by the relation (6.3). In particular, we have $\Delta n_{P, M b}(3,0)=n_{P}(2,0)=1, \Delta n_{P, M b}(3,1)=n_{P}(2,1)=2$, and $\Delta n_{P, M b}(3,2)=n_{P}(2,2)=1$. From the transformation rules obtained so far, it follows that the sources for coefficients of degree 0 for the $L_{y}=3$ Möbius strip are the coefficients of degree 0 and 2 for the corresponding cyclic strip. Adding these, we have $n_{P, M b, t o t}(3,0)=n_{P}(3,0)+n_{P}(3,2)=5$. This relation for the sum of $n_{P, M b}(3,0,+)$ and $n_{P, M b}(3,0,-)$, together with the relation $\Delta n_{P, M b}(3,0)=1$ for their difference, enables us to solve for each of these numbers, and we get $n_{P, M b}(3,0,+)=3, n_{P, M b}(3,0,-)=2$. From the transformation rule $c^{(1)} \rightarrow \pm c^{(1)}$ found previously, we infer that for the Möbius strip, $n_{P, M b, t o t}(3,1)=n_{P}(3,1)=4$. Combining this with the relation $\Delta n_{P, M b}(3,1)=2$, we solve to get $n_{P, M b}(3,1,+)=3$ and $n_{P, M b}(3,1,-)=1$. We next consider $n_{P, M b}(3,2, \pm)$. We know that $\Delta n_{P, M b}(3,2)=1$, and, given the transformation rules obtained so far, there is only one source for terms with degree coefficients of degree 2 in the chromatic polynomial for the $L_{y}=2$ Möbius strip, namely the single term with a $c^{(3)}$ coefficient. Hence, $n_{P, M b, t o t}(3,2)=1$, and so, knowing the sum and difference, we get $n_{P, M b}(3,2,+)=1$ and $n_{P, M b}(3,2,-)=0$. Since there are no sources for any higher-degree coefficients, we have $n_{P, M b}(3, d, \pm)=0$ for $d \geq 3$. Thus, we have both determined all of the numbers $n_{P, M b}(3, d, \pm)$ and the transformation rule for the coefficients of next higher degree, namely $c^{(3)} \rightarrow \pm c^{(2)}$.

Proceeding to $L_{y}=4$, we start with the knowledge of the $n_{P}(4, d)$ for the cyclic strip and of the differences $\Delta n_{P, M b}(4, d)=0$. For coefficients of degree 0 in the chromatic polynomial for the Möbius strip, the transformation rules indicate two sources, namely the coefficients with degree 0 or 2 in the corresponding cyclic strip. We shall show, it a posteriori, that these are the only sources, so we infer that $n_{P, M b, t o t}(4,0)=n_{P}(4,0)+n_{P}(4,2)=12$. Combining this with the relation $\Delta n_{P, M b}(4,0)=0$, we obtain $n_{P, M b}(4,0, \pm)=(1 / 2) n_{P, M b, t o t}(4,0)=6$. Next, for the coefficients of degree 1 , we start with those from the cyclic strip, $n_{P}(4,1)=9$. But we know that there must be another source because this is an odd number and, by itself, would give the unacceptable non-integral result for $n_{P, M b}(4,0, \pm)=(1 / 2) n_{P, M b, t o t}(4,0)$. The only possible source is the coefficients of degree 4 in the cyclic strip, so we infer the next transformation rule: $c^{(4)} \rightarrow \pm c^{(1)}$. This 
yields $n_{P, M b, t o t}(4,1)=n_{P}(4,1)+n_{P}(4,4)=10$ and hence $n_{P, M b}(4,0, \pm)=5$. Finally, for the coefficients of degree 2 in the chromatic polynomial for the $L_{y}=4$ Möbius strip, the source is the coefficients of degree 3 in the cyclic $L_{y}=4$ strip, so $n_{P, M b, t o t}(4,2)=n_{P}(4,3)=4$, whence $n_{P, M b}(4,2, \pm)=2$. Since there are no sources for any higher-degree coefficients, we have $n_{P, M b}(4, d, \pm)=0$ for $d \geq 3$. Thus again we have determined all of the numbers $n_{P, M b}(4, d, \pm)$ and also the next higher transformation rule for $c^{(4)}$.

It should now be clear how one proceeds iteratively: at each higher width $L_{y}$, one uses the previously established transformation rules, and obtains the transformation rule for the next higher degree coefficient, to determine all of the numbers $n_{P, M b}\left(L_{y}, d, \pm\right)$. This completes the proof of Theorems 5 and 6 .

Two corollaries of these theorems are

$$
\begin{gathered}
n_{P, M b}\left(L_{y}, \frac{L_{y}}{2}, \pm\right)=\frac{L_{y}}{2} \text { for even } L_{y} \\
n_{P, M b}\left(L_{y}, \frac{L_{y}+1}{2},+\right)=1, \quad n_{P, M b}\left(L_{y}, \frac{L_{y}+1}{2},-\right)=0 \text { for odd } L_{y}
\end{gathered}
$$

Values of the numbers $n_{P, M b}\left(L_{y}, d, \pm\right)$ for the first several widths, $2 \leq L_{y} \leq 10$, are given in Table 4.

Further corollaries involve ( $q$-independent) relations between the $n_{P}\left(L_{y}, d\right)$. As before for the cyclic strips, we derive these by evaluating (6.3) for $q=0,1$, and 2. For example, setting $q=0$ and using (2.17), we have (for $L_{y} \geq 2$ where Möbius strips are defined)

$$
\sum_{d=0}^{d_{\max }}(-1)^{d} \Delta n_{P, M b}\left(L_{y}, d\right)=0 \quad \text { where } \quad d_{\max }= \begin{cases}\frac{L_{y}}{2} & \text { for even } L_{y} \\ \frac{\left(L_{y}+1\right)}{2} & \text { for odd } L_{y}\end{cases}
$$

It is straightforward to derive similar relations among the $\Delta n_{P, M b}\left(L_{y}, d\right)$ by evaluating (6.3) for $q=1$ and $q=2$.

Another result pertains to the detailed structure of the chromatic polynomials for the cyclic, as compared with Möbius strips of a given width $L_{y}$, as the length $L_{x}$ gets large. As discussed in [44], because the cyclic and Möbius strips of a given width have the same number of vertices and edges, the coefficients of the leading powers of $q$ are the same. We recall this result. For $m$ greater than a minimal value ${ }^{4}$, the cyclic and Möbius strips of a given $\left(G_{s}\right)_{m}$ have the same number of vertices $n$, edges (bonds) $e$, girth $g$ (length of minimum closed circuit on $G_{s}$ ) and number $k_{g}$ of circuits of length $g$. One has $n=t_{s} m$ where $t_{s}$ depends on $G_{s}$. Writing

$$
P\left(\left(G_{s}\right), q\right)=\sum_{j=0}^{n-1}(-1)^{j} h_{n-j} q^{n-j}
$$

\footnotetext{
${ }^{4}$ For example, for $m \leq 4(m \leq 3)$ the $L_{y}=2$ cyclic (Möbius) square strips exhibit special behavior regarding $g$ and $k_{g}$; for $m$ larger than these respective values, they both have $g=4$ and $k_{g}=m$.
} 
Table 4: Table of numbers $n_{P, M b}\left(L_{y}, d, \pm\right)$ for Möbius strips of width $L_{y}$. For each $L_{y}$ value, the entries in the first and second lines are $n_{P, M b}\left(L_{y}, d,+\right)$ and $n_{P, M b}\left(L_{y}, d,-\right)$, respectively. Blank entries are zero. The last entry for each value of $L_{y}$ is the total $N_{P, L_{y}, M b, \lambda}$.

\begin{tabular}{|c|c|c|c|c|c|c|c|}
\hline \hline$L_{y} \downarrow \begin{array}{c}(d,+) \rightarrow \\
(d,-) \rightarrow\end{array}$ & $0,+$ & $1,+$ & $2,+$ & $3,+$ & $4,+$ & $5,+$ & \\
$1,-$ & $2,-$ & $3,-$ & $4,-$ & $5,-$ & $N_{P, L_{y}, M b, \lambda}$ \\
\hline \hline 2 & 1 & 1 & & & & & \\
& 1 & 1 & & & & & 4 \\
\hline 3 & 3 & 3 & 1 & & & & \\
& 2 & 1 & & & & & 10 \\
\hline 4 & 6 & 5 & 2 & & & & \\
& 6 & 5 & 2 & & & & 26 \\
\hline 5 & 16 & 15 & 8 & 1 & & & \\
& 14 & 11 & 5 & & & & 70 \\
\hline 6 & 38 & 35 & 20 & 3 & & & \\
& 38 & 35 & 20 & 3 & & & 192 \\
\hline 7 & 100 & 100 & 64 & 15 & 1 & & \\
& 96 & 91 & 56 & 11 & & & 534 \\
\hline 8 & 256 & 263 & 178 & 49 & 4 & & \\
& 256 & 263 & 178 & 49 & 4 & & 1500 \\
\hline 9 & 681 & 739 & 535 & 178 & 24 & 1 & \\
& 672 & 718 & 514 & 165 & 19 & & 4246 \\
\hline 10 & 1805 & 2027 & 1539 & 574 & 96 & 5 & \\
& 1805 & 2027 & 1539 & 574 & 96 & 5 & 12092 \\
\hline \hline
\end{tabular}


and using the results that $[13,20] h_{n-j}=\left(\begin{array}{l}e \\ j\end{array}\right)$ for $0 \leq j<g-1$ (whence $h_{n}=1$ and $h_{n-1}=e$ ) and $h_{n-(g-1)}=\left(\begin{array}{c}e \\ g-1\end{array}\right)-k_{g}$, it follows that for $m$ greater than the above-mentioned minimal value, these $h_{j}$ 's are the same for the cyclic and Möbius strips of each type $G_{s}$. For a given $G_{s}$, as $m$ increases, the $h_{n-j}$ 's for the cyclic and Möbius strips become equal for larger $j$. Our relations (6.7)-(6.9) make clear which coefficients $c_{G_{s}\left(L_{y}\right), j}$ in (1.10), and hence which coefficients $h_{G_{s}\left(L_{y}\right), j}$ in (6.19), remain equal for the cyclic versus Möbius strips.

\section{Determination of the numbers $n_{Z, M b}\left(L_{y}, d, \pm\right)$ for Möbius Strips of the Square Lattice}

In this section we generalize the calculations of the previous section to the full Potts model partition function on Möbius strips of the square lattice. (The restriction to the square lattice will be implicit in the notation.) Let $n_{Z, M b}\left(L_{y}, d, \pm\right)$ denote the number of terms $\lambda_{Z, L_{y}, j}$ in the expression (1.9) for $Z\left(L_{y} \times L_{x}, F B C_{y}, T P B C_{x}, q, v\right)$ with coefficients $c_{Z, L_{y}, M b, j}= \pm c^{(d)}$, respectively, where $M b$ denotes Möbius. The notation $c_{Z, L_{y}, M b, j}$ reflects the fact that these coefficients are independent of $L_{x}$. The sums and differences for each $d$ are defined as

$$
n_{Z, M b, t o t}\left(L_{y}, d\right)=n_{Z, M b}\left(L_{y}, d,+\right)+n_{Z, M b}\left(L_{y}, d,-\right)
$$

and

$$
\Delta n_{Z, M b}\left(L_{y}, d\right)=n_{Z, M b}\left(L_{y}, d,+\right)-n_{Z, M b}\left(L_{y}, d,-\right) .
$$

We first have the two theorems

Theorem 8.

$$
C_{Z, L_{y}, M b} \equiv \sum_{j=1}^{N_{Z, L_{y}, \lambda}} c_{Z, L_{y}, M b, j}=\sum_{d=0}^{d_{m} a x} \Delta n_{Z, M b}\left(L_{y}, d\right) c^{(d)}= \begin{cases}q^{L_{y} / 2} & \text { for even } L_{y} \\ q^{\left(L_{y}+1\right) / 2} & \text { for odd } L_{y}\end{cases}
$$

Theorem 9. For the Möbius strips of the square lattice,

$$
\begin{gathered}
n_{Z, M b}\left(L_{y}, 0, \pm\right)=\frac{1}{2}\left[n_{Z}\left(L_{y}, 0\right)+n_{Z}\left(L_{y}, 2\right) \pm n_{Z}\left(\frac{L_{y}}{2}, 0\right)\right] \quad \text { for } \quad L_{y} \text { even } \\
n_{Z, M b}\left(L_{y}, d, \pm\right)=\frac{1}{2}\left[n_{Z}\left(L_{y}, 2 d-1\right)+n_{Z}\left(L_{y}, 2 d+2\right) \pm n_{Z}\left(\frac{L_{y}}{2}, d\right)\right] \\
\text { for } L_{y} \text { even and } 1 \leq d \leq \frac{L_{y}}{2} \\
n_{Z, M b}\left(L_{y}, 0, \pm\right)=\frac{1}{2}\left[n_{Z}\left(L_{y}, 0\right)+n_{Z}\left(L_{y}, 2\right) \pm n_{Z}\left(\frac{L_{y}+1}{2}, 0\right)\right] \text { for } L_{y} \text { odd }
\end{gathered}
$$




$$
\begin{gathered}
n_{Z, M b}\left(L_{y}, d, \pm\right)=\frac{1}{2}\left[n_{Z}\left(L_{y}, 2 d-1\right)+n_{Z}\left(L_{y}, 2 d+2\right) \pm n_{Z}\left(\frac{L_{y}+1}{2}, d\right)\right] \\
\quad \text { for } L_{y} \text { odd } \text { and } 1 \leq d \leq \frac{L_{y}+1}{2} \\
n_{Z, M b}\left(L_{y}, d, \pm\right)=0 \text { for } d>\left[\frac{L_{y}+1}{2}\right]
\end{gathered}
$$

The proofs of these theorems are analogous to those for the analogous Theorems 5 and 7 for chromatic polynomials for Möbius strips of the square lattice.

Two corollaries of these theorems are

$$
\begin{gathered}
n_{Z, M b}\left(L_{y}, \frac{L_{y}}{2},+\right)=L_{y}, \quad n_{Z, M b}\left(L_{y}, \frac{L_{y}}{2},-\right)=L_{y}-1 \text { for even } L_{y} \\
n_{Z, M b}\left(L_{y}, \frac{L_{y}+1}{2},+\right)=1, \quad n_{Z, M b}\left(L_{y}, \frac{L_{y}+1}{2},-\right)=0 \quad \text { for odd } L_{y}
\end{gathered}
$$

Values of the numbers $n_{Z, M b}\left(L_{y}, d, \pm\right)$ for the first several widths, $2 \leq L_{y} \leq 10$, are given in Table 5 .

Two relations among the $n_{Z, M b}\left(L_{y}, d\right)$ can be derived as before, by evaluating the sum (7.3) for $q=0$ and $q=1$. For example, from the evaluation for $q=0$ we have (for $L_{y} \geq 2$ )

$$
\sum_{d=0}^{d_{\max }}(-1)^{d}\left(\Delta n_{Z, M b}\left(L_{y}, d\right)\right)=0 \quad \text { where } \quad d_{\max }= \begin{cases}\frac{L_{y}}{2} & \text { for even } L_{y} \\ \frac{\left(L_{y}+1\right)}{2} & \text { for odd } L_{y}\end{cases}
$$

It is straightforward to obtain a corresponding relation via the evaluation of (7.3) for $q=1$.

\section{Some Examples}

Since some of the notation above is complicated, it is worthwhile to illustrate our general calculations with specific exact solutions. These will also be useful in a discussion of the special cases of the infinite-temperature limit of the Potts model and the zero-temperature limit of the Potts ferromagnet below.

\subsection{Chromatic Polynomials}

For the $L_{y}=3$ cyclic strip of the square lattice, $N_{P, L_{y}=3, \lambda}=10$ and, with the abbreviation $s q 3 F P$ for $s q, L_{y}=3, F B C_{y}, P B C_{x}$, the chromatic polynomial is [42]

$$
P\left(s q, 3 \times L_{x}, F B C_{y}, P B C_{x}, q\right)=\sum_{j=1}^{10} c_{s q 3 F P, j}\left(\lambda_{s q 3 F P, j}\right)^{L_{x}}
$$


Table 5: Table of numbers $n_{Z, M b}\left(L_{y}, d, \pm\right)$ for Möbius strips of width $L_{y}$. For each $L_{y}$ value, the entries in the first and second lines are $n_{Z, M b}\left(L_{y}, d,+\right)$ and $n_{Z, M b}\left(L_{y}, d,-\right)$, respectively. Blank entries are zero. The last entry for each value of $L_{y}$ is the total $N_{Z, L_{y}, M b, \lambda}$.

\begin{tabular}{|c|c|c|c|c|c|c|c|}
\hline \hline$L_{y} \downarrow \begin{array}{c}(d,+) \rightarrow \\
(d,-) \rightarrow \rightarrow\end{array}$ & $\begin{array}{c}0,+ \\
0,-\end{array}$ & $\begin{array}{c}1,+ \\
1,-\end{array}$ & $\begin{array}{c}2,+ \\
2,-\end{array}$ & $\begin{array}{c}3,+ \\
3,-\end{array}$ & $\begin{array}{c}4,+ \\
4,-\end{array}$ & $\begin{array}{c}5,+ \\
5,-\end{array}$ & $N_{Z, L_{y}, M b, \lambda}$ \\
\hline \hline 2 & 2 & 2 & & & & & \\
& 1 & 1 & & & & & 6 \\
\hline 3 & 6 & 6 & 1 & & & & \\
& 4 & 3 & & & & & 20 \\
\hline 4 & 18 & 16 & 4 & & & & \\
& 16 & 13 & 3 & & & & 70 \\
\hline 5 & 61 & 54 & 20 & 1 & & & \\
& 56 & 45 & 15 & & & & 252 \\
\hline 6 & 206 & 180 & 80 & 6 & & & \\
& 201 & 171 & 75 & 5 & & & 924 \\
\hline 7 & 722 & 651 & 335 & 42 & 1 & & \\
& 708 & 623 & 315 & 35 & & & 3432 \\
\hline 8 & 2542 & 2360 & 1336 & 224 & 8 & & \\
& 2528 & 2332 & 1316 & 217 & 7 & & 12870 \\
\hline 9 & 9082 & 8766 & 5367 & 1148 & 72 & 1 & \\
& 9040 & 8676 & 5292 & 1113 & 63 & & 48620 \\
\hline 10 & 32644 & 32668 & 21270 & 5432 & 480 & 10 & \\
& 32602 & 32578 & 21195 & 5397 & 471 & 9 & 184756 \\
\hline \hline
\end{tabular}


where

$$
\begin{gathered}
\lambda_{s q 3 F P, 1}=-1 \\
\lambda_{s q 3 F P, 2}=q-1 \\
\lambda_{s q 3 F P, 3}=q-2 \\
\lambda_{s q 3 F P, 4}=q-4 \\
\lambda_{s q 3 F P, 5}=-(q-2)^{2} \\
\lambda_{s q F P,(6,7)}=\frac{1}{2}\left[(q-2)\left(q^{2}-3 q+5\right) \pm\left\{\left(q^{2}-5 q+7\right)\left(q^{4}-5 q^{3}+11 q^{2}-12 q+8\right)\right\}^{1 / 2}\right]
\end{gathered}
$$

and $\lambda_{s q F P, j}, j=8,9,10$, are the roots of the cubic equation

$$
\begin{aligned}
& \xi^{3}+\left(2 q^{2}-9 q+12\right) \xi^{2}+\left(q^{4}-10 q^{3}+36 q^{2}-56 q+31\right) \xi \\
& -(q-1)\left(q^{4}-9 q^{3}+29 q^{2}-40 q+22\right)=0 .
\end{aligned}
$$

The coefficients are

$$
\begin{gathered}
c_{s q 3 F P, 1}=c^{(3)} \\
c_{s q 3 F P, j}=c^{(2)} \text { for } j=2,3,4 \\
c_{s q 3 F P, j}=c^{(1)} \text { for } j=5,8,9,10 \\
c_{s q 3 F P, j}=c^{(0)}=1 \quad \text { for } j=6,7 .
\end{gathered}
$$

For the numbers $n_{P}\left(L_{y}, d\right)$, one sees that $n_{P}(3,0)=2, n_{P}(3,1)=4, n_{P}(3,2)=3, n_{P}(3,3)=$ 1, and the total number $N_{P, L_{y}, \lambda}=10$, in agreement with Table 1 . The exact solution for the chromatic polynomial for the $L_{y}=3$ cyclic strip of the triangular lattice, given in [46], has the same $N_{P, L_{y}, \lambda}$ and $n_{P}\left(L_{y}, d\right)$ 's, although eight of the ten $\lambda_{P, G, j}$ 's are different.

As an illustration of our results for chromatic polynomials for Möbius strips of the square lattice, we again take the $L_{y}=3$ case. The exact solution for the chromatic polynomial (with the abbreviation $s q 3 M b$ for $\left.s q, L_{y}=3, F B C_{y}, T P B C_{x}\right)$ is [44]

$$
P\left(s q, 3 \times L_{x}, F B C_{y}, T P B C_{x}, q\right)=\sum_{j=1}^{10} c_{P, s q 3 M b, j}\left(\lambda_{P, s q 3 M b, j}\right)^{L_{x}}
$$

where $\lambda_{P, s q 3 M b, j}=\lambda_{P, s q 3 F P, j} \forall j$, in accordance with the general result (3.4) and

$$
\begin{gathered}
c_{P, s q 3 M b, 1}=c^{(2)} \\
c_{P, s q 3 M b, j}=c^{(1)} \text { for } j=8,9,10 \\
c_{P, s q 3 M b, 5}=-c^{(1)} \\
c_{P, s q 3 M b, j}=c^{(0)} \quad \text { for } j=3,6,7
\end{gathered}
$$




$$
c_{P, s q 3 M b, j}=-c^{(0)} \quad \text { for } j=2,4 .
$$

Thus, for the numbers $n_{P}\left(L_{y}, d, \pm\right)$, we have $n_{P}(3,0,+)=3, n_{P}(3,0,-)=2, n_{P}(3,1,+)=3$, $n_{P}(3,1,-)=1, n_{P}(3,2,+)=1$, and $n_{P}(3,2,-)=0$, with $n_{P}(3, d, \pm)=0$ for $d \geq 3$, in agreement with Table 4.

The fact that the coefficients in the chromatic polynomial for the Möbius strip of the triangular lattice are not, in general, polynomials in $q$, was shown via the exact solution for the lowest width, $L_{y}=2$ in [42] (with $L_{x}=m$ ):

$$
\begin{gathered}
P\left(\text { tri }, 2 \times L_{x}, F B C_{y}, T P B C_{x}, q\right)=-1+\left[(q-2)^{2}\right]^{m}-\frac{(q-1)(q-3)}{\sqrt{9-4 q}}\left[\left(\lambda_{t 2 M b, 3}\right)^{m}-\left(\lambda_{t 2 M b, 4}\right)^{m}\right] \\
=-1+(q-2)^{2 m}-2^{1-m}(q-1)(q-3) \sum_{s=0}^{[(m-1) / 2]}\left(\begin{array}{c}
m \\
2 s+1
\end{array}\right)(5-2 q)^{m-2 s-1}(9-4 q)^{s}
\end{gathered}
$$

where

$$
\lambda_{t 2 M b,(3,4)}=\frac{1}{2}[5-2 q \pm \sqrt{9-4 q}] .
$$

As we have discussed in [46], this can be attributed to the feature that the Möbius strip of the triangular lattice has a seam, and hence is not translationally homogeneous in the longitudinal direction, in contrast to the Möbius strip of the square lattice, which is translationally homogeneous in the longitudinal direction.

\subsection{Potts Model Partition Function}

As an illustration for the full Potts model partition function, we recall the calculation of this function for the $L_{y}=2$ cyclic strip of the square lattice. Here, for $N_{Z, L_{y}, \lambda}$, we have [15, 16] $N_{Z, 2, \lambda}=6$, and (with the shorthand $s q 2 F P$ for $s q, L_{y}=2, F B C_{y}, P B C_{x}$ )

$$
Z\left(s q, 2 \times L_{x}, F B C_{y}, P B C_{x}, q, v\right)=\sum_{j=1}^{6} c_{Z, s q 2 F P, j}\left(\lambda_{Z, s q 2 F P, j}\right)^{L_{x}}
$$

where

$$
\begin{gathered}
\lambda_{Z, s q 2 F P, 1}=v^{2} \\
\lambda_{Z, s q 2 F P, 2}=v(v+q) \\
\lambda_{Z, s q 2 F P,(3,4)}=\frac{v}{2}\left[q+v(v+4) \pm\left(v^{4}+4 v^{3}+12 v^{2}-2 q v^{2}+4 q v+q^{2}\right)^{1 / 2}\right] \\
\lambda_{Z, s q 2 F P,(5,6)}=\frac{1}{2}\left(T_{S 12} \pm \sqrt{R_{S 12}}\right)
\end{gathered}
$$

with

$$
\begin{gathered}
T_{S 12}=v^{3}+4 v^{2}+3 q v+q^{2} \\
R_{S 12}=v^{6}+4 v^{5}-2 q v^{4}-2 q^{2} v^{3}+12 v^{4}+16 q v^{3}+13 q^{2} v^{2}+6 q^{3} v+q^{4} .
\end{gathered}
$$


and

$$
\begin{gathered}
c_{Z, s q 2 F P, 1}=c^{(2)} \\
c_{Z, s q 2 F P, j}=c^{(1)} \text { for } j=2,3,4 \\
c_{Z, s q 2 F P, j}=c^{(0)} \text { for } j=5,6 .
\end{gathered}
$$

Thus, for the individual numbers, $n_{Z}(2,0)=2, n_{Z}(2,1)=3, n_{Z}(2,2)=1$, and $n_{Z}(2, d)=0$ for $d \geq 3$, in agreement with Table 3 .

As an example of our general results for the structure of the Potts model partition function on Möbius strips of the square lattice, we display the exact solution for width $L_{y}=2[15,16]$ (with the abbreviation $s q 2 M b$ for $s q, L_{y}=2, F B C_{y}, T P B C_{x}$ ):

$$
Z\left(s q, 2 \times L_{x}, F B C_{y}, T P B C_{x}, q, v\right)=\sum_{j=1}^{6} c_{Z, s q 2 M b, j}\left(\lambda_{Z, s q 2 M b, j}\right)^{L_{x}}
$$

where $\lambda_{Z, s q 2 M b, j}=\lambda_{Z, s q 2 F P, j} \forall j$, in accord with the result (3.6) and

$$
\begin{aligned}
c_{Z, s q 2 M b, j}=c^{(1)} & \text { for } j=3,4 \\
c_{Z, s q 2 M b, 2}= & -c^{(1)} \\
c_{Z, s q 2 M b, j}=c^{(0)} & \text { for } j=5,6 \\
c_{Z, s q 2 M b, 1}= & -c^{(0)}
\end{aligned}
$$

Thus, $n_{Z, M b}(2,0,+)=2, n_{Z, M b}(2,0,-)=1, n_{Z, M b}(2,1,+)=2$, and $n_{Z, M b}(2,1,+)=1$, in agreement with Table 5 .

\subsection{Other Limits}

In the text above we have given results for structural properties of the general Potts model partition function $Z(G, q, v)$ and for the special case of the zero-temperature Potts antiferromagnet, $Z(G, q, v=-1)=P(G, q)$. Another special case is infinite-temperature, i.e., $v=0$. In this case, for any graph $G$, the Potts model partition function reduces to $Z(G, q, 0)=q^{n(G)}$, as indicated above in (1.8). For the strip graphs under consideration here, the equality in (1.8) corresponds to the property that at $v=0$ all but one of the $\lambda_{Z, G, j}$ 's in eq. (1.9) vanish, and the nonvanishing $\lambda_{Z, G, j}$ necessarily has the value $q^{L_{y}}$ and the coefficient $c^{(0)}=1$, so that the right-hand side of (1.9) becomes $q^{L_{y} L_{x}}=q^{n}$. One can see this explicitly in the exact solution given above for the Potts model partition function for the $L_{y}=2$ strip of the square lattice.

Secondly, one may consider the zero-temperature limit of the Potts ferromagnet, $v \rightarrow \infty$. This is quite different from the $T=0$ limit of the Potts antiferromagnet; among other differences, the ground state entropy per site is zero for the ferromagnet rather than being nonzero as it is for the antiferromagnet with sufficiently large $q$. In this limit $T \rightarrow 0$, the partition function of the Potts ferromagnet grows like $a^{e(G)}$ where $a$ was defined in (1.3) and 
$e(G)$ was also defined in the introduction as the number of edges (bonds) in $G$. Hence, it is convenient to use the reduced partition function $Z_{r}$, defined by

$$
Z_{r}(G, q, v)=a^{-e(G)} Z(G, q, v)=u^{e(G)} Z(G, q, v)
$$

which has the finite limit $Z_{r} \rightarrow 1$ as $T \rightarrow 0$. For the cyclic or Möbius strip graphs of the lattice $\Lambda$ of interest here we thus write

$$
\begin{aligned}
Z_{r}\left(\Lambda, F B C_{y},(T) P B C_{x}, q, v\right) & =u^{e(G)} \sum_{j=1}^{N_{Z, G, \lambda}} c_{Z, G, j}\left(\lambda_{Z, G, j}\right)^{L_{x}} \\
& \equiv \sum_{j=1}^{N_{Z, G, \lambda}} c_{Z, G, j}\left(\lambda_{Z, G, j, u}\right)^{L_{x}}
\end{aligned}
$$

where as before we use the shorthand notation $G=\Lambda, F B C_{y},(T) P B C_{x}$, and

$$
\lambda_{Z, G, j, u}=u^{e(G) / L_{x}} \lambda_{Z, G, j} .
$$

For example, for cyclic or Möbius strips of the square lattice, $e=\left(2 L_{y}-1\right) L_{x}$, so the prefactor in (8.3.3) is $u^{2 L_{y}-1}$, while for the corresponding strips of the triangular lattice, $e=\left(3 L_{y}-2\right) L_{x}$, so the prefactor is $u^{3 L_{y}-2}$. For the $L_{y}=1$ circuit graph,

$$
\lambda_{Z, C, 1, u}=1-u, \quad \lambda_{Z, C, 2, u}=1+(q-1) u .
$$

so that both of these two $\lambda_{Z, G, j, u}$ 's remain important in the $T=0$ limit. In contrast, for the $L_{y}=2$ cyclic or Möbius strips of the square and triangular lattices, our exact solutions in $[16,47]$ show that, of the six $\lambda_{Z, G, j, u}$ 's, two are finite and nonzero for $u \rightarrow 0$ while the other four vanish. For example, for the cyclic/Möbius strip of the square lattice, in the vicinity of the zero-temperature point $u=0$, one has [16]

$$
\begin{gathered}
\lambda_{Z, s q 2 F P, 1, u}=u-2 u^{2}+u^{3} \\
\lambda_{Z, s q 2 F P, 2, u}=u+(q-2) u^{2}+(1-q) u^{3}
\end{gathered}
$$

and the Taylor series expansions

$$
\begin{gathered}
\lambda_{Z, s q 2 F P, 3, u}=1-u^{2}+2(q-2) u^{3}+O\left(u^{4}\right) \\
\lambda_{Z, s q 2 F P, 4, u}=u+(q-4) u^{2}+(7-3 q) u^{3}+O\left(u^{4}\right) \\
\lambda_{Z, s q 2 F P, 5, u}=1+(q-1) u^{2}\left[1+4 u+O\left(u^{2}\right)\right] \\
\lambda_{Z, s q 2 F P, 6, u}=u+2(q-2) u^{2}+\left(q^{2}-7 q+7\right) u^{3}+O\left(u^{4}\right) .
\end{gathered}
$$

Hence,

$$
\lim _{u \rightarrow 0} \frac{\lambda_{Z, s q 2 F P, j, u}}{\lambda_{Z, s q 2 F P, 3, u}}=\lim _{v \rightarrow \infty} \frac{\lambda_{Z, s q 2 F P, j}}{\lambda_{Z, s q 2 F P, 3}}=\left\{\begin{array}{ll}
1 & \text { for } j=5 \\
0 & \text { for } j=1,2,4,6
\end{array} .\right.
$$

In this sense, one can define an effective, reduced, $N_{Z, G, \lambda}$, and this has the value 2 , somewhat analogous to the value $N_{P, G, \lambda}=4$ for the $T=0$ limit of the Potts antiferromagnet (1.10); however, a difference is that when one reinserts the prefactor in (8.3.2) to get back the actual partition function, all of the six $\lambda_{Z, G, j}$ 's do contribute. 


\section{Coefficients for Other Strip Graphs}

\subsection{Strips with Torus or Klein Bottle Boundary Conditions}

Like the cyclic and Möbius strips, the strips with torus or Klein bottle boundary conditions have periodic or twisted periodic longitudinal boundary conditions, respectively. We first give two theorems for the sum of the coefficients for these types of strip graphs (which are the analogues of the theorems yielding eqs. (3.10) and (6.3) for the cyclic and Möbius strips). We have

Theorem 10. The sum of the coefficients in (1.16) for a strip of the square or triangular lattice of width $L_{y}$ and $\left(P B C_{y}, P B C_{x}\right)=$ torus boundary conditions is (independent of $L_{x}$ )

$$
C_{P, L_{y}, \text { torus }}=\sum_{j=1}^{N_{P, L_{y}, \text { torus }, \lambda}} c_{P, L_{y}, \text { torus }, j}=P\left(C_{L_{y}}, q\right)
$$

where the chromatic polynomial of the circuit graph $C_{n}$ is

$$
P\left(C_{n}, q\right)=(q-1)^{n}+(q-1)(-1)^{n} .
$$

Proof. This follows by noting that the transverse slice of the strip graphs of the square and triangular lattices of width $L_{y}$ is the circuit graph $C_{L_{y}}$, and the number of ways of proper colorings of this graph is given by the chromatic polynomial in (9.1.2).

Theorem 11. The sum of the coefficients in (1.16) for a strip of the square or triangular lattice of width $L_{y}$ is (independent of $\left.L_{x}\right)$ and $\left(P B C_{y}, T P B C_{x}\right)=$ Klein bottle $(\mathrm{KB})$ boundary conditions is

$$
C_{P, L_{y}, K B}=\sum_{j=1}^{N_{P, L_{y}, K B, \lambda}} c_{P, L_{y}, K B, j}=0
$$

Proof. This follows by observing that this sum corresponds to the proper coloring of an effective graph (defined as in our study of Möbius strips) formed from the transverse slice of the strip, and in this case the effective graph always involves at least one loop, i.e. an edge that connects a vertex to itself (specifically, the effective graph involves one loop if $L_{y}$ is odd and two loops if $L_{y}$ is even). But the chromatic polynomial of a graph with a loop vanishes identically.

Theorems 10 and 11 generalize the observations made for these sums of coefficients for the exact solutions of the chromatic polynomials of the $L_{y}=3$ torus and Klein bottle graphs of the square lattice in [45] and [46] to arbitrary $L_{y}$ values where these graphs are defined, i.e. $L_{y} \geq 3$. 
On the basis of the exact solutions for the chromatic polynomials for the width $L_{y}=$ 3 strips of the square [45] and triangular [46] lattices with $\left(P B C_{y}, P B C_{x}\right)=$ torus and $\left(P B C_{y}, T P B C_{x}\right)=$ Klein bottle boundary conditions, we can make several further remarks. First, since it was found that $N_{P, G, \lambda}=8$ for $G=s q\left(3 \times L_{x}, P B C_{y}, P B C_{x}\right)$ but the different value, $N_{P, G, \lambda}=11$ for $\operatorname{tri}\left(3 \times L_{x}, P B C_{y}, P B C_{x}\right)$, these strips behave fundamentally differently than the cyclic and Möbius strips of these two lattices, for which the value of $N_{P, G, \lambda}$ was the same for a given $L_{y}$. Secondly, the exact solutions in $[45,46]$ show that the coefficients that enter in the chromatic polynomial for the $L_{y}=3$ strips of the square and triangular lattice with torus or Klein bottle boundary conditions are polynomials in $q$ but are not of the form $c^{(d)}=U_{2 d}\left(\frac{\sqrt{q}}{2}\right)$. Indeed, in contrast to the situation for cyclic and Möbius squarelattice strips and cyclic triangular-lattice strips, the coefficient of degree $d$ is not unique, i.e., there can be more than one type of coefficient of a given degree $d$ in $q$. For example, for the $L_{y}=3$ square-lattice strip with torus boundary conditions, the coefficients that are quadratic functions of $q$ are $q(q-3),(1 / 2) q(q-3),(q-1)(q-2)$, and $(1 / 2)(q-1)(q-2)$. Another difference, again illustrated by the chromatic polynomial for the $L_{y}=3$ strips of the square and triangular lattice is that the coefficients can have zeros outside the range $0<q<4$, in contrast to the $c^{(d)}$. For example, one term in the chromatic polynomial for the $L_{y}=3$ strip with toroidal boundary conditions is $\left(q^{3}-6 q^{2}+8 q-1\right)(-1)^{L_{x}}$ for the square lattice [45] and $(1 / 3)(q-1)\left(q^{2}-5 q+3\right)(-2)^{L_{x}}$ for the triangular lattice [46]; the first coefficient has a zero at $q \simeq 4.11$ and the other coefficient has a zero at $q \simeq 4.30$. Given that the exact solutions for the chromatic polynomials on torus graphs in $[45,46]$ show that the coefficients, which are multiplicities of eigenvalues of the coloring matrix, are not of the form $c^{(d)}$ for strip graphs of the square and triangular lattices, it is not clear to us how to relate this with the remark in [29] that certain related eigenvalue multiplicities $d_{r}$ for torus boundary conditions are of the form $\sin (r \theta / 2) / \sin (\theta / p)$ where $p$ is 1 (2) if $r$ is even (odd) and $\theta$ satisfies $(2.25)$.

The exact solutions for the chromatic polynomial in [45] for the square lattice and in [46] for the triangular lattice showed that if one starts with torus boundary conditions, cuts the tube graph and reglues the ends with reversed orientation to form Klein bottle boundary conditions, the number of $\lambda_{P, G, j}$ 's does change (is reduced):

$$
N_{P, \Lambda, L_{y} \times L_{x}, P B C_{y}, P B C_{x}} \neq N_{P, \Lambda, L_{y} \times L_{x}, P B C_{y}, T P B C_{x}} .
$$

Specifically, it was found that $[45,15,46]$ while

$$
N_{P, s q, 3 \times L_{x}, P B C_{y}, P B C_{x}, \lambda}=8, \quad N_{P, t r i, 3 \times L_{x}, P B C_{y}, P B C_{x}, \lambda}=11
$$

in contrast,

$$
N_{P, s q, 3 \times L_{x}, P B C_{y}, T P B C_{x}, \lambda}=N_{P, t r i, 3 \times L_{x}, P B C_{y}, P B C_{x}, \lambda}=5 .
$$

Thus, at least for this width, if one uses Klein bottle boundary conditions, then the value of $N_{P, G, \lambda}$ is the same, namely five, for both square and triangular strips. As is evident from the exact solutions $[45,46]$, the five coefficients are the same, although the $\lambda_{P, G, j}$ 's are different. These coefficients are

$$
\left\{1, q-1,-(q-1),-\frac{(q-1)(q-2)}{2}, \frac{q(q-3)}{2}\right\}
$$


Since the coloring matrix applies directly for the lattice strip graphs with torus boundary conditions, eq. (1.18) applies for the chromatic polynomials of these graphs.

\subsection{Strips with Free Longitudinal Boundary Conditions}

Previously, exact solutions for chromatic polynomials on strip graphs of regular lattices with $\left(F B C_{y}, F B C_{x}\right)=$ open boundary conditions were given in [33, 40]; in these cases, as discussed before, the coefficients $c_{P, G, j}$ are not, in general, polynomials in $q$. This can be seen immediately from eqs. (2.14) or (2.19) of [40]. An analogous statement holds for the full Potts model partition functions on these open strips [15, 16, 47]. This property can be understood as a consequence of the fact that the chromatic polynomial is not the trace of an $m$ 'th power of the coloring matrix for these strips, but rather is given by

$$
P(G, q)=\left\langle c\left|\mathcal{T}^{m}\right| c^{\prime}\right\rangle
$$

where $c$ and $c^{\prime}$ denote colorings of the ends of the strip. A similar statement holds for the full Potts partition function $Z(G, q, v)$ in terms of the corresponding matrix $\mathcal{T}_{Z}$.

As discussed before [36, 41, 42, 44, 15, 46], for a strip graph of some lattice with given transverse boundary conditions, the term $\lambda_{P, G, j}$ that is dominant in the physical region of $q$ (including sufficiently positive integers and denoted as region $R_{1}$ in our previous work [14]) is independent of the longitudinal boundary conditions. (The same applies for the $\lambda_{Z, G, j}$ in $Z(G, q, v)$ that is dominant in the physical paramagnetic phase [15, 16, 47].) In particular, this means that the $\lambda_{P, G, j}$ that is dominant in region $R_{1}$ is the same for the cyclic (or Möbius) and open strip graphs of a given lattice type $\Lambda$, thereby establishing a certain connection between these cyclic (or Möbius) and open strip graphs. Similarly, it means that the $\lambda_{P, G, j}$ that is dominant in region $R_{1}$ is the same for the torus and Klein bottle strips of this lattice $\Lambda$ on the one hand, and the cylindrical strip graphs on the other. Now it was also shown [15] that for graphs with periodic or twisted periodic boundary conditions, the coefficient of this dominant term is unity. If this term is a root $r_{\ell, s}$ of an (irreducible) algebraic equation of degree $d_{\ell}$, then the theorem on symmetric functions of roots of an algebraic equation implies that for the cyclic strips, these roots $r_{\ell, s}$ appear in $P(G, q)$ in the form of the sum $\sum_{s=1}^{d_{\ell}} r_{\ell, s}^{L_{x}}$, i.e., they all have the coefficient unity [44]. This sets an upper bound on $d_{\ell}\left(\Lambda, L_{y}\right)$, namely

$$
n_{P}\left(L_{y}, 0\right) \geq d_{\ell}\left(\Lambda, L_{y}\right)
$$

where we have included the dependent of $d_{\ell}$ on the lattice type $\Lambda$ and the strip width, and we recall from eq. (3.4) that $d_{\ell}\left(\Lambda, L_{y}\right)$ is the same for the cyclic and Möbius strips of a given lattice $\Lambda$. For cyclic and Möbius strips of the square lattice, $d_{\ell}(s q, 1)=1, d_{\ell}(s q, 2)=1$ [18], $d_{\ell}(s q, 3)=2$ [42], and $d_{\ell}(s q, 4)=3$ [48], while for the cyclic and Möbius strips of the triangular lattice, $d_{\ell}(\operatorname{tri}, 2)=1$ [42] and $d_{\ell}(\operatorname{tri}, 3)=2, d_{\ell}(\operatorname{tri}, 4)=4$ [46]. This information is summarized in tables given in $[46,48]$ along with related results. Our calculations above yield, for the relevant values of $n_{P}\left(L_{y}, 0\right)$ (see Table 1 ): $n_{P}(1,0)=n_{P}(2,0)=1, n_{P}(3,0)=2$, and $n_{P}(4,0)=4$. Hence, evidently, the inequality (9.2.2) is realized as an equality for the 
triangular strips and the square strips for $L_{y}=1,2,3$, but as a strict inequality for the $L_{y}=4$ square strips. Further, for the cases that we studied, we found that

$$
d_{\ell}\left(\Lambda, L_{y}\right)_{c y c ., M b}=d_{\ell}\left(\Lambda, L_{y}\right)_{\text {open }}
$$

where $d_{\ell}\left(\Lambda, L_{y}\right)_{\text {open }} \equiv d_{\ell}\left(\Lambda, F B C_{y}, F B C_{x}, L_{y}\right)$. When we obtained the results in the present paper for $n_{P}\left(L_{y}, d\right)$, we noticed the interesting connection that for the cyclic and Möbius strips of the triangular lattice, for which (9.2.2) holds as an equality, it is also true that one has the fourfold equality

$$
n_{P}\left(L_{y}, 0\right)=d_{\ell}\left(\operatorname{tri}, L_{y}\right)_{c y c .,} \text { Mb. }=d_{\ell}\left(\operatorname{tri}, L_{y}\right)_{\text {open }}=N_{P, \operatorname{tri}\left(L_{y}\right), \text { open }, \lambda}
$$

i.e., the number of $\lambda_{P, G, j}$ 's with coefficient $c^{(0)}=1$ in the chromatic polynomial for the cyclic or Möbius strips of the triangular lattice, which was equal to the degree of the algebraic equation one of whose roots was the dominant $\lambda_{P, G, j}$ in $R_{1}$, was also equal to the total number of terms in the chromatic polynomial for the open strip of the triangular lattice with the same width. We confirmed eq. (9.2.4) for widths $L_{y}=2,3,4$, based on the exact solutions in $[33,42,46]$. In terms of the generating function formalism of $[33,34,40]$, the denominator $\mathcal{D}$ of the generating function, as a polynomial in the auxiliary variable, has the maximal degree, $n_{P}\left(L_{y}, 0\right)$, allowed by (9.2.2) for these open strips of the triangular lattice but does not, in general, for the open strips of the square lattice. As we noted in $[46,48]$, this difference in behavior is related to the fact that the Möbius strips of the square and triangular lattices are different; in particular, the Möbius strip of the triangular lattice involves algebraic nonpolynomial coefficients. Further, even for the strips of the square lattice, where the first two terms of (9.2.4) are not, in general, equal (as our solutions for chromatic polynomials for $L_{y}=4$ showed), we still found

$$
d_{\ell}\left(s q, L_{y}\right)_{c y c ., M b .}=N_{P, s q\left(L_{y}\right), o p e n, \lambda} .
$$

That is, we find that the equality (9.2.5) holds for cyclic or Möbius and open strips of the square lattice for $1 \leq L_{y} \leq 4$, as well as for the corresponding strips of the triangular lattice with $2 \leq L_{y} \leq 4$; in particular, it holds even when $n_{P}\left(L_{y}, 0\right)$ is not equal to (is greater than) $d_{\ell}\left(\Lambda, L_{y}\right)$. For reference, for open strips of the triangular lattice with $L_{y} \geq 2$, one can infer from calculations in [29] that $N_{Z, \operatorname{tri}\left(L_{y}\right), \text { open, } \lambda}=C_{L_{y}}$; furthermore, $N_{P, \operatorname{tri}\left(L_{y}\right), \text { open, },}=M_{L_{y}-1}$ [49]. Another generalization would be that for triangular strips, the value of the degree in eq. (9.2.3) satisfies $d_{\ell}\left(\text { tri }, L_{y}\right)_{c y c ., M b}=d_{\ell}\left(\text { tri }, L_{y}\right)_{\text {open }}=M_{L_{y}-1}$. This would require that, in terms of the generating function [33], the denominator $\mathcal{D}$ does not factorize.

Previously, coloring matrix methods have been applied to the honeycomb $(h c)$ lattice to derive rigorous bounds on $W[23,24]$. In the present context of strips, we observe that, in addition to the elementary result $N_{P, h c, L_{y}=2, \text { open, } \lambda}=d_{\ell}\left(h c, L_{y}=2\right)_{\text {open }}=1$, our exact solutions have yielded the results

$$
N_{P, h c, L_{y}=3, \text { opend }}=d_{\ell}\left(h c, L_{y}=3\right)_{\text {open }}=3
$$

from [33] and

$$
N_{P, h c, L_{y}=2, c y c ., M b_{., \lambda}}=d_{\ell}\left(h c, L_{y}=2\right)_{c y c ., M b}=1
$$

from [41]. In view of eq. (9.2.6), for this strip of the honeycomb lattice, $n_{P}\left(L_{y}=3,0\right) \neq$ $d_{\ell}\left(h c, L_{y}=3\right)_{\text {open }}$, in contrast to the case with the square and triangular lattice strips. 


\subsection{Strips with Cylindrical Boundary Conditions}

Exact solutions for chromatic polynomials for cylindrical strips, i.e. with boundary conditions of the form $\left(P B C_{y}, F B C_{x}\right)$ of the square and triangular lattices were presented in $[34,36]$, and, again, the coefficients are, in general, not polynomials in $q$, for the same reason as in the case of the open strips. And again, the same statement holds for the full Potts model partition function. (Recent calculations of chromatic polynomials for wider strips with open and cylindrical boundary conditions include [46, 48, 49].)

\section{Some General Geometric Identities}

In this section we present some useful identities between Potts model partition functions on different types of lattice strips that follow from basic geometrical considerations. We have applied these as checks in our previous calculations. Consider a family of strip graphs of the square or triangular lattice with fixed width $L_{y}$ and arbitrary length $L_{x}$ with some set of longitudinal $(x)$ and transverse $(y)$ boundary conditions, denoted as $B C=\left(B C_{y}, B C_{x}\right)$. By the transfer matrix argument given in [16], it follows that the Potts model partition function for this strip is of the form

$$
Z\left(\Lambda, L_{y} \times L_{x}, B C, q, v\right)=\sum_{j=1}^{N_{Z, \Lambda, L_{y}, B C}} c_{Z, \Lambda, L_{y}, B C, j}\left(\lambda_{Z, \Lambda, L_{y}, B C, j}\right)^{L_{x}}
$$

We recall [16] that the total number of terms, $N_{Z, \Lambda, L_{y}, B C}$, the coefficients $c_{Z, \Lambda, L_{y}, B C, j}$, and the $\lambda_{Z, \Lambda, L_{y}, B C, j}$ 's are independent of $L_{x}$. In our previous works, we have studied the $L_{x} \rightarrow \infty$ behavior of these Potts model partition functions. However, clearly, for fixed $L_{y}$ and $L_{x}$, by switching the $x$ and $y$ axes, one can equivalently view the strip as the length $-L_{y}$ member of a family of strip graphs with fixed width $L_{x}$ and length $L_{y} \cdot{ }^{5}$ This yields a number of useful identities. We proceed to describe these

\subsection{Strips with $\left(F B C_{y}, F B C_{x}\right)$}

For strips of the square and triangular lattices with $\left(F B C_{y}, F B C_{x}\right)$ (open) boundary conditions, the interchange of the $x$ and $y$ axes leaves the boundary conditions the same, and one gets the following identity (where we write $F_{y} F_{x} \equiv\left(F B C_{y}, F B C_{x}\right)$ to save space)

$$
\sum_{j=1}^{N_{Z, \Lambda, L_{y}, F_{y}, F_{x}}} c_{Z, \Lambda, L_{y}, F_{y}, F_{x}, j}\left(\lambda_{Z, \Lambda, L_{y}, F_{y}, F_{x}, j}\right)^{L_{x}}=\sum_{j=1}^{N_{Z, \Lambda, L_{x}, F_{x}, F_{y}}} c_{Z, \Lambda, L_{x}, F_{x}, F_{y}, j}\left(\lambda_{Z, \Lambda, L_{x}, F_{x}, F_{y}, j}\right)^{L_{y}}
$$

\footnotetext{
${ }^{5}$ Note that for other lattices such simple relations do not, in general, hold. For example, if one considers strips of the honeycomb lattice, constructed as a brick lattice, then rotating a open horizontal $L_{y} \times L_{x}$ strip of bricks by $90^{\circ}$, one does not get an equivalent $L_{x} \times L_{y}$ strip of horizontally oriented bricks.
} 
Taking $T=0$ for the Potts antiferromagnet, one obtains the corresponding identity for chromatic polynomials

$$
\sum_{j=1}^{N_{P, \Lambda, L_{y}, F_{y}, F_{x}}} c_{P, \Lambda, L_{y}, F_{y}, F_{x}, j}\left(\lambda_{P, \Lambda, L_{y}, F_{y}, F_{x}, j}\right)^{L_{x}}=\sum_{j=1}^{N_{P, \Lambda, L_{x}, F_{y}, F_{x}}} c_{P, \Lambda, L_{x}, F_{x}, F_{y}, j}\left(\lambda_{P, \Lambda, L_{x}, F_{x}, F_{y}, j}\right)^{L_{y}}
$$

Using the results in [33], one can construct various illustrations of this. One of the simplest is to take the open strip of the square lattice with width $L_{y}=2$, for which $N_{P, s q, 2, F B C_{y}, F B C_{x}}=1$ and $P\left(s q, 2 \times L_{x}, F B C_{y}, F B C_{x}, q\right)=q(q-1)\left(q^{2}-3 q+3\right)^{L_{x}-1}$. For $L_{x}=3$, this chromatic polynomial must be equal to that for the width $L_{y}=3$ open strip (which has $N_{P, s q, 3, F B C_{y}, F B C_{x}}=2$ ) with length $L_{x}=2$, for which (using [33] and eq. (2.15) of [40]) for general $L_{x}$,

$$
\begin{aligned}
& P\left(s q, 3 \times L_{x}, F B C_{y}, F B C_{y}, q\right)=\frac{1}{\left(\lambda_{s q 3 F F, 1}-\lambda_{s q 3 F F, 2}\right)} \times \\
& {\left[\left(A_{s q 3 F F, 0} \lambda_{s q 3 F F, 1}+A_{s q 3 F F, 1}\right)\left(\lambda_{s q 3 F F, 1}\right)^{L_{x}-2}-\left(A_{s q 3 F F, 0} \lambda_{s q 3 F F, 2}+A_{s q 3 F F, 1}\right)\left(\lambda_{s q 3 F F, 2}\right)^{L_{x}-2}\right]}
\end{aligned}
$$

where

$$
\begin{gathered}
A_{s q 3 F F, 0}=q(q-1)\left(q^{2}-3 q+3\right)^{2} \\
A_{s q 3 F F, 1}=-q(q-1)^{3}\left(q^{3}-6 q^{2}+13 q-11\right)
\end{gathered}
$$

and

$$
\lambda_{s q 3 F F,(1,2)}=\frac{1}{2}\left[(q-2)\left(q^{2}-3 q+5\right) \pm\left[\left(q^{2}-5 q+7\right)\left(q^{4}-5 q^{3}+11 q^{2}-12 q+8\right)\right]^{1 / 2}\right] .
$$

\subsection{Cyclic and Cylindrical Strips}

We have denoted strips of fixed width $L_{y}$ and arbitrary length $L_{x}$ vertices with $\left(F B C_{y}, P B C_{x}\right)$ and $\left(P B C_{y}, F B C_{x}\right)$ boundary conditions as cyclic and cylindrical, respectively. Topologically, these are the same; the distinction was made because we were, in particular, interested in the large $L_{x} \rightarrow \infty$ limit, which, for cyclic strips means that the length of a circuit goes to infinity, while for the cylindrical strips, the length of the circumference of the cylinder is fixed while its length goes to infinity. Clearly, a cyclic strip of the square or triangular lattice with a fixed width $L_{y}$ and length $L_{x}$ is the same as a cylindrical strip of the given lattice with width $L_{x}$ and length $L_{y}$. Letting $\Lambda$ denote the type of lattice, square or triangular as before, and writing

$$
Z\left(\Lambda, L_{y} \times L_{x}, F B C_{y}, P B C_{x}\right)=\sum_{j=1}^{N_{Z, \Lambda, L_{y}, F_{y}, P_{x}}} c_{Z, \Lambda, L_{y}, F_{y}, P_{x}, j}\left(\lambda_{Z, \Lambda, L_{y}, F_{y}, P_{x}, j}\right)^{L_{x}}
$$


we have the identity

$$
\sum_{j=1}^{N_{Z, \Lambda, L_{y}, F_{y}, P_{x}}} c_{Z, \Lambda, L_{y}, F_{y}, P_{x}, j}\left(\lambda_{Z, \Lambda, L_{y}, F_{y}, P_{x}, j}\right)^{L_{x}}=\sum_{j=1}^{N_{Z, \Lambda, L_{x}, P_{y}, F_{x}}} c_{Z, \Lambda, L_{x}, P_{y}, F_{x}, j}\left(\lambda_{Z, \Lambda, L_{x}, P_{y}, F_{x}, j}\right)^{L_{y}} .
$$

Again, for the special case of the $T=0$ Potts antiferromagnet, we have the resultant identity for the chromatic polynomial

$$
\sum_{j=1}^{N_{P, \Lambda, L_{y}, F_{y}, P_{x}}} c_{P, \Lambda, L_{y}, F_{y}, P_{x}, j}\left(\lambda_{P, \Lambda, L_{y}, F_{y}, P_{x}, j}\right)^{L_{x}}=\sum_{j=1}^{N_{P, \Lambda, L_{x}, P_{y}, F_{x}}} c_{P, \Lambda, L_{x}, P_{y}, F_{x}, j}\left(\lambda_{P, \Lambda, L_{x}, P_{y}, F_{x}, j}\right)^{L_{y}} .
$$

Perhaps the simplest illustration of this general identity is the relation between the chromatic polynomial for the width $L_{y}=2$ cyclic strip of the square lattice and the width $L_{y}=3$ cylindrical strip of this lattice. For the $L_{y}=2$ cyclic strip, one has $N_{P, s q, 2 \times L_{x}, F_{y}, P_{x}}=4$ and

$P\left(s q, 2 \times L_{x}, F B C_{y}, P B C_{x}, q\right)=\left(q^{2}-3 q+1\right)+(q-1)\left[(3-q)^{L_{x}}+(1-q)^{L_{x}}\right]+\left(q^{2}-3 q+3\right)^{L_{x}}$.

For the $L_{y}=3$ cylindrical strip, one has

$$
P\left(s q, 3 \times L_{x}, P B C_{y}, F B C_{x}, q\right)=q(q-1)(q-2)\left(q^{3}-6 q^{2}+14 q-13\right)^{L_{x}-1} .
$$

Thus, the identity (10.2.3) yields

$$
\begin{aligned}
& \left(q^{2}-3 q+1\right)+(q-1)\left[(3-q)^{3}+(1-q)^{3}\right]+\left(q^{2}-3 q+3\right)^{3}= \\
& q(q-1)(q-2)\left(q^{3}-6 q^{2}+14 q-13\right) .
\end{aligned}
$$

While this illustration is quite simple, more complicated cases involve identities between powers of algebraic roots of different types.

\subsection{Strips with Torus Boundary Conditions}

For a strip graph of the square or triangular lattice with $\left(P B C_{y}, P B C_{x}\right) \equiv\left(P_{y}, P_{x}\right)$ (torus) boundary conditions, the identities read

$$
\begin{gathered}
\sum_{j=1}^{N_{Z, \Lambda, L_{y}, P_{y}, P_{x}}} c_{Z, \Lambda, L_{y}, P_{y}, P_{x}, j}\left(\lambda_{Z, \Lambda, L_{y}, P_{y}, P_{x}, j}\right)^{L_{x}}=\sum_{j=1}^{N_{Z, \Lambda, L_{x}, P_{x}, P_{y}}} c_{Z, \Lambda, L_{x}, P_{x}, P_{y}, j}\left(\lambda_{Z, \Lambda, L_{x}, P_{x}, P_{y}, j}\right)^{L_{y}} \\
\sum_{j=1}^{N_{P, \Lambda, L_{y}, P_{y}, P_{x}}} c_{P, \Lambda, L_{y}, P_{y}, P_{x}, j}\left(\lambda_{P, \Lambda, L_{y}, P_{y}, P_{x}, j}\right)^{L_{x}}=\sum_{j=1}^{N_{P, \Lambda, L_{x}, P_{x}, P_{y}}} c_{P, \Lambda, L_{x}, P_{x}, P_{y}, j}\left(\lambda_{P, \Lambda, L_{x}, P_{x}, P_{y}, j}\right)^{L_{y}}
\end{gathered}
$$


The simplest illustration of this is for the family of strips of the square lattice with width $L_{y}=$ 2 , for which the torus degenerates to a cyclic strip, and one has $P\left(s q, 2 \times L_{x}, P B C_{y}, P B C_{x}, q\right)=$ $P\left(s q, 2 \times L_{x}, F B C_{y}, P B C_{x}, q\right)$, given in (10.2.4). Let us next consider the strip of the square lattice with width $L_{y}=3$ and length $L_{x}=2$. This graph is related by the identity to two other strip graphs: (i) the cyclic strip of the square lattice with $L_{y}=2$ and $L_{x}=3$ (see eq. (10.2.4)) and (ii) the equivalent cylindrical strip of the square lattice with $L_{y}=3$ and $L_{x}=2$ (see eq. (10.2.5)). Indeed, when one evaluates the exact solution given in [45] for $L_{x}=2$, one finds that it is equal to the right-hand side of eq. (10.2.6).

\section{Conclusions}

In this paper, using a general formula, in terms of Chebyshev polynomials of the second kind, for the coefficients that occur in the $q$-state Potts model partition function on cyclic strips of the square and triangular lattices and on Möbius strips of the square lattice, together with general formulas for sums of these coefficients, we have determined the number, $n_{Z}\left(L_{y}, d\right)$, of $\lambda_{Z, G, j}$ 's with coefficient $c^{(d)}$ in $Z(G, q, v)$ and the total number, $N_{Z, G, \lambda}$, for these strips. Results are also given for the analogous numbers $n_{P}\left(L_{y}, d\right)$ and $N_{P, L_{y}, \lambda}$ for the zero-temperature Potts antiferromagnet partition functions, i.e., chromatic polynomials for these strips. The results for the total numbers, $N_{Z, L_{y}, \lambda}$ and $N_{P, L_{y}, \lambda}$, apply for both cyclic and Möbius strips of both the square and triangular lattices. Among other connections, we found that $n_{P}\left(L_{y}, 0\right)=n_{P}\left(L_{y}-1,1\right)=M_{L_{y}-1}$, the Motzkin number; $n_{Z}\left(L_{y}, 0\right)=C_{L_{y}}$, the Catalan number; the exact expression for $N_{P, L_{y}, \lambda}$ in eq. (5.1.2); and the relations $N_{P, L_{y}, \lambda}=2 N_{D A, s q, L_{y}}$; and $N_{Z, L_{y}, \lambda}=2 N_{D A, t r i, L_{y}}$, where $N_{D A, \Lambda, n}$ denotes the number of directed lattice animals on the lattice $\Lambda$. We also found the asymptotic growths $N_{Z, L_{y}, \lambda} \sim L_{y}^{-1 / 2} 4^{L_{y}}$ and $N_{P, L_{y}, \lambda} \sim L_{y}^{-1 / 2} 3^{L_{y}}$ as $L_{y} \rightarrow \infty$. Some commens about other lattice strips were made. In addition, we presented some useful general geometric identities for Potts model partition functions.

Acknowledgment: We thank Lee-Peng Teo for helpful discussions. The research of R. S. was supported in part by the NSF grant PHY-9722101 and at Brookhaven by the DOE contract DE-AC02-98CH10886. ${ }^{6}$

\footnotetext{
${ }^{6}$ Accordingly, the U.S. government retains a non-exclusive royalty-free license to publish or reproduce the published form of this contribution or to allow others to do so for U.S. government purposes.
} 


\section{Appendix}

\subsection{Connection Between Potts Model Partition Function and Tutte Polynomial}

The Potts model partition function $Z(G, q, v)$ is related to the Tutte polynomial $T(G, x, y)$ as follows. The graph $G$ has vertex set $V$ and edge set $E$, denoted $G=(V, E)$. A spanning subgraph $G^{\prime}$ is defined as a subgraph that has the same vertex set and a subset of the edge set: $G^{\prime}=\left(V, E^{\prime}\right)$ with $E^{\prime} \subseteq E$. The Tutte polynomial of $G, T(G, x, y)$, is then given by [7]-[9]

$$
T(G, x, y)=\sum_{G^{\prime} \subseteq G}(x-1)^{k\left(G^{\prime}\right)-k(G)}(y-1)^{c\left(G^{\prime}\right)}
$$

where $k\left(G^{\prime}\right), e\left(G^{\prime}\right)$, and $n\left(G^{\prime}\right)=n(G)$ denote the number of components, edges, and vertices of $G^{\prime}$, and

$$
c\left(G^{\prime}\right)=e\left(G^{\prime}\right)+k\left(G^{\prime}\right)-n\left(G^{\prime}\right)
$$

is the number of independent circuits in $G^{\prime}$ (sometimes called the co-rank of $G^{\prime}$ ). Note that the first factor can also be written as $(x-1)^{r(G)-r\left(G^{\prime}\right)}$, where

$$
r(G)=n(G)-k(G)
$$

is called the rank of $G$. The graphs $G$ that we consider here are connected, so that $k(G)=1$. Now let

$$
x=1+\frac{q}{v}
$$

and

$$
y=a=v+1
$$

so that $q=(x-1)(y-1)=(x-1) v$. Then

$$
Z(G, q, v)=(x-1)^{k(G)}(y-1)^{n(G)} T(G, x, y) .
$$

There is also a connection with the Whitney rank polynomial, $R(G, \xi, \eta)$, defined as $[4,10]$

$$
R(G, \xi, \eta)=\sum_{G^{\prime} \subseteq G} \xi^{r\left(G^{\prime}\right)} \eta^{c\left(G^{\prime}\right)}
$$

where the sum is again over spanning subgraphs $G^{\prime}$ of $G$. Then

$$
T(G, x, y)=(x-1)^{r(G)} R\left(G, \xi=(x-1)^{-1}, \eta=y-1\right)
$$

and

$$
Z(G, q, v)=q^{n(G)} R\left(G, \xi=\frac{v}{q}, \eta=v\right)
$$


Note that the chromatic polynomial is a special case of the Tutte polynomial:

$$
P(G, q)=q^{k(G)}(-1)^{k(G)+n(G)} T(G, x=1-q, y=0)
$$

(recall eq. (1.6)).

For a recursive family of graphs, such as the strip graphs considered in this paper, comprised of $m$ repetitions of a basic subgraph, the Tutte polynomial has the form [16]

$$
T(G, x, y)=\sum_{j=1}^{N_{T, G, \lambda}} c_{T, G, j}\left(\lambda_{T, G, j}\right)^{m}
$$

where, from (12.1.6), one has the relation

$$
\lambda_{T, G, j}=v^{-L_{y}} \lambda_{Z, G, j}
$$

so that

$$
N_{T, G, \lambda}=N_{Z, G, \lambda} .
$$

It is convenient to extract a common factor from the coefficients:

$$
c_{T, G, j} \equiv \frac{\bar{c}_{T, G, j}}{x-1} .
$$

Of course, although the individual terms contributing to the Tutte polynomial are thus rational functions of $x$ rather than polynomials in $x$, the full Tutte polynomial is a polynomial in both $x$ and $y$. Given the relation (12.1.6), if one defines $n_{T}\left(L_{y}, d\right)$ as the number of terms $\lambda_{T, L_{y}, j}$ in $T\left(L_{y}, q, v\right)$ that have as their reduced coefficient $\bar{c}_{T, L_{y}, j}=c^{(d)}$, then these are the same numbers:

$$
n_{T}\left(L_{y}, d\right)=n_{Z}\left(L_{y}, d\right) .
$$

Thus, Tables 3 and 5 apply equally to the structure of the Tutte polynomials for the cyclic and Möbius strips of the square and triangular lattices.

For a given graph $G=(V, E)$, at certain special values of the arguments $x$ and $y$, the Tutte polynomial $T(G, x, y)$ yields quantities of basic graph-theoretic interest [9]-[11]. We recall some definitions: a spanning subgraph was defined at the beginning of the paper; a tree is a connected graph with no cycles; a forest is a graph containing one or more trees; and a spanning tree is a spanning subgraph that is a tree. We recall that the graphs $G$ that we consider are connected. Then the number of spanning trees of $G, N_{S T}(G)$, is

$$
N_{S T}(G)=T(G, 1,1) \text {, }
$$

the number of spanning forests of $G, N_{S F}(G)$, is

$$
N_{S F}(G)=T(G, 2,1),
$$

the number of connected spanning subgraphs of $G, N_{C S S G}(G)$, is

$$
N_{C S S G}(G)=T(G, 1,2) \text {, }
$$

and the number of spanning subgraphs of $G, N_{S S G}(G)$, is

$$
N_{S S G}(G)=T(G, 2,2) \text {. }
$$

These connections have been used in [79],[80]. 


\subsection{Determinants of Coloring Matrices}

In this section we list the determinants of coloring matrices for some lattice strips. For the chromatic polynomial this is

$$
\operatorname{det} T_{P}(G)=\prod_{j=1}^{\mathcal{N}} \lambda_{P, G, j}=\prod_{j=1}^{N_{P, G, \lambda}}\left(\lambda_{P, G, j}\right)^{c_{P, G, j}}
$$

For the Potts model partition function the analogous determinant is

$$
\operatorname{det} T_{Z}(G)=\prod_{j=1}^{\mathcal{N}_{\mathcal{Z}}} \lambda_{Z, G, j}=\prod_{j=1}^{N_{Z, G, \lambda}}\left(\lambda_{Z, G, j}\right)^{c_{Z, G, j}}
$$

Let us define the shorthand notation

$$
D_{P}(G)=\operatorname{det} T_{P}(G), \quad D_{Z}(G)=\operatorname{det} T_{Z}(G) .
$$

For the products of eigenvalues contributing to (1.10) for the chromatic polynomials and (1.9) for the Potts model partition function for cyclic and Möbius strips of the square lattice we find

$$
D_{P}\left(s q, L_{y}=1, F B C_{y}, P B C_{x}\right)=D_{P}(\{C\}, q)=(-1)^{q-1}(q-1)
$$

(where $\{C\}$ refers to the circuit graph) For the $L_{y}=2$ cyclic and Möbius strips of the square lattice, from [18],

$$
D_{P}\left(s q, L_{y}=2, F B C_{y}, P B C_{x}\right)=[(3-q)(1-q)]^{q-1}\left(q^{2}-3 q+3\right)
$$

and

$$
D_{P}\left(s q, L_{y}=2, F B C_{y}, T P B C_{x}\right)=(3-q)^{q-1}(1-q)^{-(q-1)}\left(q^{2}-3 q+3\right) .
$$

For the $L_{y}=3$ cyclic and Möbius strips of the square lattice, from [42],

$$
\begin{aligned}
& D_{P}\left(s q, L_{y}=3, F B C_{y}, P B C_{x}\right)=(-1)^{(q-2)\left(q^{2}-3 q+1\right)}(q-1)^{(q-1)^{2}}(q-2)^{q^{2}-q-1} \times \\
& (q-4)^{q^{2}-3 q+1}\left(q^{3}-6 q^{2}+13 q-11\right)\left(q^{4}-9 q^{3}+29 q^{2}-40 q+22\right)^{q-1}
\end{aligned}
$$

and

$$
\begin{aligned}
& D_{P}\left(s q, L_{y}=3, F B C_{y}, T P B C_{x}\right)=(-1)^{q^{2}-4 q+2}(q-1)^{q-1}(q-2)^{-(2 q-3)} \times \\
& (q-4)^{-1}\left(q^{3}-6 q^{2}+13 q-11\right)\left(q^{4}-9 q^{3}+29 q^{2}-40 q+22\right)^{q-1}
\end{aligned}
$$

The corresponding determinants for $L_{y}=4$ can be obtained from [48] but are quite lengthy, so we do not list them here. 
For the $D_{Z}$ determinants we have

$$
D_{Z}\left(s q, L_{y}=1, F B C_{y}, P B C_{x}\right)=D_{Z}(\{C\}, q)=v^{q-1}(q+v)
$$

and, from [16],

$$
D_{Z}\left(s q, L_{y}=2, F B C_{y}, P B C_{x}\right)=v^{2 q(q-1)}(v+1)^{q}(v+q)^{2 q}
$$

and

$$
D_{Z}\left(s q, L_{y}=2, F B C_{y}, T P B C_{x}\right)=v^{2(q-1)}(v+1)^{q}(v+q)^{2} .
$$

Note that, except for the case of the circuit graph, $s q, L_{y}=1, P B C_{x}$, for the value $v=-1$, where the Potts model partition function reduces to the chromatic polynomial, $Z(G, q, v=$ $-1)=P(G, q)$, some eigenvalues in the product contributing to $Z$ vanish, and hence this product vanishes. If one extracts these vanishing eigenvalues, then, of course, the rest yield the same product as for $P(G, q)$.

For cyclic strips of the triangular lattice we have, from [42]

$$
D_{P}\left(\text { tri }, L_{y}=2, F B C_{y}, P B C_{x}\right)=(q-2)^{2 q}
$$

and from $[46,47]$,

$$
\begin{gathered}
D_{P}\left(\operatorname{tri}, L_{y}=3, F B C_{y}, P B C_{x}\right)=(-1)^{(q-2)\left(q^{2}-3 q+1\right)}(q-2)^{q(2 q-1)}(q-3)^{q(q-1)} \\
D_{Z}\left(t r i, L_{y}=2, F B C_{y}, P B C_{x}\right)=v^{2 q(q-1)}(v+1)^{2 q}(v+q)^{2 q} .
\end{gathered}
$$

The determinant $D_{P}\left(\operatorname{tri}, L_{y}=4, F B C_{y}, P B C_{x}\right)$ can be calculated from our exact solution in [46]; however, it is sufficiently lengthy that we do not include it here. The analogous determinants for the Möbius strips of the triangular lattice can also be calculated from the exact solutions that we have given [42, 46]; however, they are more complicated, since some coefficients are algebraic, rather than polynomial, functions of $q$.

For the strip graphs of the square and triangular lattices with $\left(P B C_{y}, P B C_{x}\right)=$ torus and $\left(P B C_{y}, T P B C_{x}\right)=$ Klein bottle boundary conditions, we have, from the exact solutions in [45] and [46],

$$
\begin{aligned}
& D_{P}\left(s q, L_{y}=3, P B C_{y}, P B C_{x}\right)=(-1)^{q^{3}-6 q^{2}+11 q-4}(q-1)^{\frac{(q-1)(q-2)}{2}} \times \\
& (q-2)^{q^{2}+q-4}(q-4)^{(q-1)(q-2)}(q-5)^{\frac{q(q-3)}{2}}\left(q^{2}-7 q+13\right)^{q-1} \times \\
& \left(q^{3}-6 q^{2}+14 q-13\right)
\end{aligned}
$$

and

$$
\begin{aligned}
D_{P}\left(s q, L_{y}=3, P B C_{y}, T P B C_{x}\right)= & (q-1)^{-\frac{(q-1)(q-2)}{2}}(q-5)^{\frac{q(q-3)}{2}}\left(q^{2}-7 q+13\right)^{q-1} \times \\
& \left(q^{3}-6 q^{2}+14 q-13\right) .
\end{aligned}
$$




\subsection{Some Other Coloring Matrix Results for Lattice Strip Graphs}

Since the trace of a coloring matrix vanishes (recall (1.18)), the sum of eigenvalues, each multiplied by its multiplicity also vanishes, as indicated in eq. (1.19), for the cyclic and torus strips, for which (1.14) applies directly. Using coloring methods, we obtain the following general formulas for sums for other types of strips. These agree with our previous exact solutions in $[42,46,48]$.

$$
\begin{gathered}
\sum_{j=1}^{N_{P, s q\left(L_{y}\right), M b, \lambda}} c_{P, s q\left(L_{y}\right), M b, j} \lambda_{P, s q\left(L_{y}\right), M b, j}= \begin{cases}q(q-1)\left(q^{2}-3 q+3\right)^{\frac{L_{y}}{2}-1} & \text { for even } L_{y} \\
0 & \text { for odd } L_{y}\end{cases} \\
\sum_{j=1}^{N_{P, \operatorname{tri}\left(L_{y}\right), M b, \lambda}} c_{P, \operatorname{tri}\left(L_{y}\right), M b, j} \lambda_{P, \operatorname{tri}\left(L_{y}\right), M b, j}=0 \quad \forall L_{y} \geq 2 .
\end{gathered}
$$

The equations also apply to the corresponding strips with Klein bottle (KB) boundary conditions, with $M b \rightarrow K B$, and $L_{y} \geq 3$. The polynomial $q^{2}-3 q+3$ in (12.3.1) is $D_{4}$ in our previous notation, where

$$
D_{k}=\sum_{s=0}^{k-2}(-1)^{s}\left(\begin{array}{c}
k-1 \\
s
\end{array}\right) q^{k-2-s}
$$

From the exact solutions for the chromatic polynomials in [42], we have, for the cyclic strip of the kagomé lattice consisting of a succession of hexagons with two interleaved triangles per hexagon of arbitrary length (denoted as having width $L_{y}=2$ in [42]) we have

$$
\sum_{j=1}^{6} c_{P, k a g 2, j} \lambda_{P, k a g 2, j}=q(q-1)^{2}(q-2)^{2}
$$

Additional families of graphs are provided by the homeomorphic expansions of the cyclic and Möbius $L_{y}=2$ strips of the square lattice, in which one adds $k-2$ degree- 2 vertices to the upper and lower horizontal edges of each square, for $k \geq 3$. These may be viewed as strips of $p$-sided polygons, with $p=2 k$, with each successive pair of polygons sharing one edge. For $k=2$ and $k=3$, these families are the $L_{y}=2$ cyclic and Möbius strips of the square and honeycomb lattice, respectively. (In the latter case, the honeycomb lattice is represented as a brick lattice with the bricks oriented horizontally.) Following [41], we denote the cyclic (cyc) and Möbius (Mb) strips of this type, of length $L_{x}=m$ as $(C h)_{k, m, c y c}$ and $(C h)_{k, m, M b}$, where $C h$ denotes "chain". Exact solutions for the chromatic polynomials of these families were given in [41]. From the construction of these graphs, it is clear that $\sum_{j} c_{P,(C h)_{k, c y c, j}}=q(q-1)$ and $\sum_{j} c_{P,(C h)_{k, M b, j}}=0$ hold, as in eqs. (3.10) and (6.3). For the other sums (with $\lambda_{(C h), k, c y c, j}=\lambda_{(C h), k, M b, j}$ ), we have

$$
\sum_{j=1}^{4} c_{(C h)_{k, c y c, j}} \lambda_{(C h)_{k, c y c, j}}=q^{2}-3 q+1+2(-1)^{k+1}(q-1) D_{k+1}+D_{2 k}
$$


where $D_{k}$ was defined in (12.3.3), and

$$
\sum_{j=1}^{4} c_{(C h)_{k, M b, j}} \lambda_{(C h)_{k, M b, j}}=-1+2(-1)^{k}(q-1) D_{k}+D_{2 k} .
$$

For $k=2$, the sum (12.3.5) is zero, as in (1.18), and the sum (12.3.6) has the value $q(q-1)$ as in the $L_{y}=2$ special case of (12.3.1). As an example of the values for higher $-p$ polygonal strips, for $k=3$, i.e., the strip of the honeycomb lattice, (12.3.5) has the value $q(q-1)^{3}$ and (12.3.6) has the value $q(q-1)(q-2)^{2}$.

For the full Potts model partition functions, from our exact solutions in $[16,47]$ we find

$$
\begin{gathered}
\sum_{j=1}^{6} c_{Z, s q 2, c y c, j} \lambda_{Z, s q 2, c y c, j}=q(v+1)^{2}(v+q) \\
\sum_{j=1}^{6} c_{Z, s q 2, M b, j} \lambda_{Z, s q 2, M b, j}=q\left(v^{3}+3 v^{2}+3 v+q\right) \\
\sum_{j=1}^{6} c_{Z, t r i 2, c y c, j} \lambda_{Z, t r i 2, c y c, j}=q(v+1)^{2}\left(v^{2}+2 v+q\right) \\
\sum_{j=1}^{6} c_{Z, t r i 2, M b, j} \lambda_{Z, t r i 2, M b, j}=q(v+1)\left(v^{3}+3 v^{2}+3 v+q\right) .
\end{gathered}
$$

As is evident, for the special case $v=-1$ where the Potts model partition function reduces to the chromatic polynomial, these equations reduce to their analogues for the respective chromatic polynomials.

In terms of the Tutte polynomials, these formulas read

$$
\begin{gathered}
\sum_{j=1}^{6} c_{T, s q 2, c y c, j} \lambda_{T, s q 2, c y c, j}=x y^{2} \\
\sum_{j=1}^{6} c_{T, s q 2, M b, j} \lambda_{T, s q 2, M b, j}=x+y+y^{2}
\end{gathered}
$$

(which is the Tutte polynomial for the graph known as the "thick link" with three edges, the planar dual to the circuit graph $C_{3}$ )

$$
\sum_{j=1}^{6} c_{T, t r i 2, c y c, j} \lambda_{T, t r i 2, c y c, j}=(x+y) y^{2}
$$


and

$$
\sum_{j=1}^{6} c_{T, t r i 2, M b, j} \lambda_{T, t r i 2, M b, j}=y\left(x+y+y^{2}\right) .
$$

\section{References}

[1] R. B. Potts, Proc. Camb. Phil. Soc. 48106 (1952).

[2] F. Y. Wu, Rev. Mod. Phys. 54 (1982) 235.

[3] G. D. Birkhoff, Ann. of Math. 14 (1912) 42.

[4] H. Whitney, Ann. of Math. 33 (1932) 688.

[5] P. W. Kasteleyn and C. M. Fortuin, J. Phys. Soc. Jpn. 26 (1969) (Suppl.) 11.

[6] C. M. Fortuin and P. W. Kasteleyn, Physica 57 (1972) 536.

[7] W. T. Tutte, Can. J. Math. 6 (1954) 80.

[8] W. T. Tutte, J. Combin. Theory 2 (1967) 301.

[9] W. T. Tutte, "Chromials", in Lecture Notes in Math. v. 411 (1974) 243; Graph Theory, vol. 21 of Encyclopedia of Mathematics and Applications (Addison-Wesley, Menlo Park, 1984).

[10] N. L. Biggs, Algebraic Graph Theory (2nd ed., Cambridge Univ. Press, Cambridge, 1993).

[11] D. J. A. Welsh, Complexity: Knots, Colourings, and Counting, London Math. Soc. Lect. Note Ser. 186 (Cambridge University Press, Cambridge, 1993).

[12] R. C. Read, J. Combin. Theory 4 (1968) 52.

[13] R. C. Read and W. T. Tutte, "Chromatic Polynomials", in Selected Topics in Graph Theory, 3, eds. L. W. Beineke and R. J. Wilson (Academic Press, New York, 1988.).

[14] R. Shrock and S.-H. Tsai, Phys. Rev. E55 (1997) 5165.

[15] R. Shrock, in the Proceedings of the 1999 British Combinatorial Conference, BCC99 (July, 1999); Physica A 281, 221 (2000).

[16] R. Shrock, Physica A, in press.

[17] E. H. Lieb, Phys. Rev. Lett. 18 (1967) 692; Phys. Rev. 162 (1967) 162. 
[18] N. L. Biggs, R. M. Damerell, and D. A. Sands, J. Combin. Theory B 12 (1972) 123.

[19] D. A. Sands, D. A., Ph.D. Thesis, Univ. of London, 1972 (unpublished).

[20] G. H. Meredith, J. Combin. Theory B 13 (1972) 14.

[21] N. L. Biggs and G. H. Meredith, J. Combin. Theory B20 (1976) 5

[22] N. L. Biggs, Bull. London Math. Soc. 9 (1976) 54.

[23] R. Shrock and S.-H. Tsai, Phys. Rev. E55 (1997) 6791.

[24] R. Shrock and S.-H. Tsai, Phys. Rev. E56 (1997) 2733, 4111.

[25] S. Beraha, J. Kahane, and N. Weiss, J. Combin. Theory B 27 (1979) 1.

[26] S. Beraha, J. Kahane, and N. Weiss, J. Combin. Theory B 28 (1980) 52.

[27] R. C. Read, in Proc. 3rd Caribbean Conf. on Combin. and Computing (1981).

[28] R. C. Read, in Proc. 5th Caribbean Conf. on Combin. and Computing (1988).

[29] R. J. Baxter, J. Phys. A 20 (1987) 5241.

[30] D. Klein and W. Seitz, in MATH/CHEM/COMP 1988, Studies in Physical and Theoretical Chemistry, vol. 63, p. 155.

[31] R. C. Read and G. F. Royle, in Graph Theory, Combinatorics, and Applications (Wiley, NY, 1991), vol. 2, p. 1009.

[32] R. J. Baxter, S. B. Kelland, and F. Y. Wu, J. Phys. A 9, 397 (1976).

[33] M. Roček, R. Shrock, and S.-H. Tsai, Physica A252 (1998) 505.

[34] M. Roček, R. Shrock, and S.-H. Tsai, Physica A259 (1998) 367.

[35] R. Shrock and S.-H. Tsai, Phys. Rev. E56 (1997), 3935.

[36] R. Shrock and S.-H. Tsai, Phys. Rev. E58 (1998) 4332, cond-mat/9808057.

[37] R. Shrock and S.-H. Tsai, J. Phys. A 31 (1998) 9641.

[38] R. Shrock and S.-H. Tsai, Physica A265 (1999) 186.

[39] A. Sokal, Combin. Prob. Comput., in press.

[40] R. Shrock and S.-H. Tsai, Physica A259 (1998) 315.

[41] R. Shrock and S.-H. Tsai, J. Phys. A Lett. 32 (1999) L195; J. Phys. A 32 (1999) 5053.

[42] R. Shrock and S.-H. Tsai, Phys. Rev. E60 (1999) 3512; Physica A 275 (2000) 429. 
[43] N. L. Biggs, LSE report LSE-CDAM-99-03 (May 1999), to appear.

[44] R. Shrock, Phys. Lett. A261 (1999) 57.

[45] N. L. Biggs and R. Shrock, J. Phys. A (Letts) 32, L489 (1999).

[46] S.-C. Chang and R. Shrock, Stony Brook preprints YITP-SB-99-50,58 (Oct. 1999); cond-mat/0004129.

[47] S.-C. Chang and R. Shrock, Physica A, in press (cond-mat/0004181)

[48] S.-C. Chang and R. Shrock, cond-mat/0004161

[49] J. Salas and A. Sokal, cond-mat/0004330,

[50] H. N. V. Temperley and E. H. Lieb, Proc. Roy. Soc. A322 (1971) 251.

[51] R. J. Baxter, Exactly Solved Models (Academic Press, New York, 1982).

[52] P. P. Martin, J. Phys. A 20 (1987) L539, L601.

[53] P. P. Martin, Potts Models and Related Problems in Statistical Mechanics (World Scientific, Singapore, 1991).

[54] I. Gradshtein and I. Ryzhik, Tables of Integrals, Series, and Products (Academic Press, New York, 1980), eq. (8.940.2).

[55] J. Riordan, Combinatorial Identities (Wiley, New York, 1968).

[56] A. A. Migdal, Zh. Eksp. Teor. Fiz. 69 (1975) 1457 (JETP 42 (1975) 743); A. M. Polyakov, Phys. Lett. B59 (1975) 79.

[57] E. Brézin and J. Zinn-Justin, Phys. Rev. B14 (1976) 3110.

[58] W. Bardeen, B. W. Lee, and R. Shrock, Phys. Rev. D14 (1976) 985.

[59] V. Matveev and R. Shrock, Phys. Lett. A204 (1995) 353.

[60] M. P. M. den Nijs, J. Phys. A 12 (1979) 1857.

[61] B. Nienhuis, E. K. Riedel, and M. Schick, J. Phys. A 13, L189 (1980).

[62] R. B. Pearson, Phys. Rev. B22, 2579 (1980).

[63] T. Motzkin, Bull. Amer. Math. Soc. 54 (1948) 352.

[64] R. Donaghey and L. W. Shapiro, J. Combin. Theory, A 23 (1977) 291.

[65] M. Aigner, Europ. J. Combin. 19 (1998) 663. 
[66] R. P. Stanley, Enumerative Combinatorics (Cambridge University Press, Cambridge, 1999), v. 2.

[67] F. R. Bernhart, Disc. Math. 204 (1999) 73.

[68] N. J. A. Sloane and S. Plouffe, The Encyclopedia of Integer Sequences (Academic Press, New York, 1995).

[69] N. J. A. Sloane, The On-Line Encyclopedia of Integer Sequences, http://www.research.att.com/ ${ }^{\sim}$ njas/sequences/.

[70] D. Dhar, M. Phani, and M. Barma, J. Phys. A15 (1982), L279.

[71] D. Dhar, Phys. Rev. Lett. 49 (1982) 959; ibid. 51 (1983) 853.

[72] R. J. Baxter, J. Phys. A 13 (1981) L61.

[73] H. E. Stanley, S. Redner, and Z. R. Yang, J. Phys. A 15 (1982) L569; J. Cardy, J. Phys. A 15 (1982) 593.

[74] A. Conway, R. Brak, and A. J. Guttmann, J. Phys. A 26 (1993) 3085.

[75] A. J. Guttmann, Phys. Rev. Lett. 76 (1996) 344.

[76] M. Bousquet-Mélou, Disc. Math. 180 (1998) 73.

[77] D. Arrowsmith and J. Essam, Phys. Rev. Lett. 65 (1990) 3068.

[78] F. Y. Wu et al., Phys. Rev. Lett. 76, 173 (1996).

[79] F. Y. Wu, J. Phys. A 10 (1977) L113; W. J. Tzeng and F. Y. Wu, Lett. Appl. Math., in press (cond-mat/0001408).

[80] R. Shrock and F. Y. Wu, J. Phys. A, in press (cond-mat/0004341). 